\title{
Lax representations with non-removable parameters and integrable hierarchies of PDEs via exotic cohomology of symmetry algebras
}

\author{
Oleg I. Morozov \\ Faculty of Applied Mathematics, AGH University of Science and Technology, \\ Al. Mickiewicza 30, Cracow 30-059, Poland, \\ and \\ Institute of Control Sciences of Russian Academy of Sciences, \\ Profsoyuznaya 65, Moscow 117997, Russia
}

\begin{abstract}
This paper develops the technique of constructing Lax representations for PDEs via non-central extensions generated by non-triivial exotic 2-cocycles of their contact symmetry algebras. We show that the method is applicable to the Lax representations with non-removable spectral parameters. Also we demonstrate that natural extensions of the symmetry algebras produce the integrable hierarchies associated to their PDEs.

Keywords: exotic cohomology, Maurer-Cartan forms, symmetries of differential equations, Lax representations 2000 MSC: 58H05, 58J70, 35A30, 37K05, 37K10

Subject Classification: integrable PDEs, symmetries of PDEs, cohomology of Lie algebras
\end{abstract}

\section{Introduction}

Lax representations, also known as zero-curvature representations, Wahlquist-Estabrook prolongation structures, inverse scattering transformations, or differential coverings [20, 21] , are a key feature of integrable partial differential equations (PDEs). A number of important techniques for studying integrable PDEs such as Bäcklund transformations, Darboux transformations,

Email address: morozov@agh.edu.pl (Oleg I. Morozov) 
recursion operators, nonlocal symmetries, and nonlocal conservation laws, are based on Lax representations. Lax representations with non-removable (spectral) parameter are of special interest in the theory of integrable PDEs, see, e.g., [1, 12, 13, 41]. The challenging unsolved problem in this theory is to find conditions that are formulated in inherent terms of a PDE under study and ensure existence of a Lax representation. Recently, an approach to this problem has been proposed in [34, 35], where it was shown that for some PDEs their Lax representations can be inferred from the second exotic cohomology group of the contact symmetry algebras of the PDEs.

The present paper provides an important supplement to the technique of [34, 35]. Namely, we show that Lax representations with non-removable parameters arise naturally from non-central extensions of the symmetry algebras generated by nontrivial second exotic cohomology groups. We consider here four equations: the hyper-CR equation for Einstein-Weyl structures [23, 28, 39, 15]

$$
u_{y y}=u_{t x}+u_{y} u_{x x}-u_{x} u_{x y}
$$

the reduced quasi-classical self-dual Yang-Mills equation [17]

$$
u_{y z}=u_{t x}+u_{y} u_{x x}-u_{x} u_{x y}
$$

the four-dimensional equation

$$
u_{z z}=u_{t x}+u_{z} u_{x y}-u_{x} u_{y z}
$$

introduced in [7], and the four-dimensional Martínez Alonso-Shabat equation [27]

$$
u_{t y}=u_{z} u_{x y}-u_{y} u_{x z}
$$

Equations (2) and (44) are related by a Bäcklund transformation, [22], while their contact symmetry algebras are not isomorphic. Furthermore, they are symmetry reductions of the quasi-classical self-dual Yang-Mills equation [24, 25, 2, 7]

$$
u_{y z}=u_{t x}+u_{x} u_{z s}-u_{z} u_{x s}
$$

The 3-dimensional reduction of equation (3) defined by substitution for $u_{t}=0$ produces the universal hierarchy equation [26, 27]

$$
u_{z z}=u_{z} u_{x y}-u_{x} u_{y z}
$$


therefore we refer equation (3) to as the four-dimensional universal hierarchy equation.

The Lax representations with non-removable parameters $\lambda$ for equations (11) - (41) are defined by systems

$$
\begin{aligned}
& \left\{\begin{array}{l}
v_{t}=\left(\lambda^{2}-\lambda u_{x}-u_{y}\right) v_{x}, \\
v_{y}=\left(\lambda-u_{x}\right) v_{x},
\end{array}\right. \\
& \left\{\begin{array}{l}
v_{t}=\lambda v_{y}-u_{y} v_{x}, \\
v_{z}=\left(\lambda-u_{x}\right) v_{x},
\end{array}\right. \\
& \left\{\begin{array}{l}
v_{t}=\lambda^{2} v_{x}-\left(\lambda u_{x}+u_{z}\right) v_{y}, \\
v_{z}=\lambda v_{x}-u_{x} v_{y},
\end{array}\right.
\end{aligned}
$$

and

$$
\left\{\begin{array}{l}
v_{y}=\lambda u_{y} v_{x}, \\
v_{z}=\lambda\left(u_{z} v_{x}-v_{t}\right),
\end{array} .\right.
$$

These systems were found in [28, 39, 15], [17], [40], and [33], respectively.

The following structure distinguishes the contact symmetry algebras for equations (11) - (4): these Lie algebras are semi-direct products $\mathfrak{g}_{\infty} \rtimes \mathfrak{g}_{\diamond}$ of an infinite-dimensional ideal $\mathfrak{g}_{\infty}$ and a non-Abelian finite-dimensional Lie algebra $\mathfrak{g}_{\diamond}$. The second exotic cohomology groups of the finite-dimensional subalgebras $\mathfrak{g}_{\diamond}$ turn out to be nontrivial for all the equations, and the corresponding nontrivial 2-cocycles produce non-central extensions of their symmetry algebras $\mathfrak{g}_{\infty} \rtimes \mathfrak{g}_{\diamond}$. We show that certain linear combinations of the Maurer-Cartan forms of the extensions define the Lax representations (7), (8), and (10), while the Lax representation (9) can be revealed via the same procedure applied twice.

The infinite-dimensional ideals $\mathfrak{g}_{\infty}$ of the symmetry algebras for the equations under consideration admit series of natural extensions $\widehat{\mathfrak{g}}_{\infty}$ that preserve the actions of the finite-dimensional Lie algebras $\mathfrak{g}_{\diamond}$ as well as the nontrivial 2-cocycles from the second exotic cohomology groups of $\mathfrak{g}_{\diamond}$. The nontrivial 2-cocycles generate non-central extensions of the Lie algebras $\widehat{\mathfrak{g}}_{\infty} \rtimes \mathfrak{g}_{\diamond}$. The Maurer-Cartan forms of the extensions provide Lax representations for integrable hierarchies associated with equations (11) - (4). Thus we show that the integrable hierachies are invariantly and intrinsically related to the equations under the study. 


\section{Preliminaries}

All considerations in this paper are local. All functions are assumed to be real-analytic.

\subsection{Symmetries and differential coverings}

The presentation in this subsection closely follows [18, 19], see also [20, 21, 42]. Let $\pi: \mathbb{R}^{n} \times \mathbb{R}^{m} \rightarrow \mathbb{R}^{n}, \pi:\left(x^{1}, \ldots, x^{n}, u^{1}, \ldots, u^{m}\right) \mapsto\left(x^{1}, \ldots, x^{n}\right)$, be a trivial bundle, and $J^{\infty}(\pi)$ be the bundle of its jets of the infinite order. The local coordinates on $J^{\infty}(\pi)$ are $\left(x^{i}, u^{\alpha}, u_{I}^{\alpha}\right)$, where $I=\left(i_{1}, \ldots, i_{n}\right)$ are multiindices, and for every local section $f: \mathbb{R}^{n} \rightarrow \mathbb{R}^{n} \times \mathbb{R}^{m}$ of $\pi$ the corresponding infinite jet $j_{\infty}(f)$ is a section $j_{\infty}(f): \mathbb{R}^{n} \rightarrow J^{\infty}(\pi)$ such that $u_{I}^{\alpha}\left(j_{\infty}(f)\right)=$ $\frac{\partial^{\# I} f^{\alpha}}{\partial x^{I}}=\frac{\partial^{i_{1}+\ldots+i_{n}} f^{\alpha}}{\left(\partial x^{1}\right)^{i_{1}} \ldots\left(\partial x^{n}\right)^{i_{n}}}$. We put $u^{\alpha}=u_{(0, \ldots, 0)}^{\alpha}$. Also, we will simplify notation in the following way, e.g., in the case of $n=4, m=1$ : we denote $x^{1}=t, x^{2}=x, x^{3}=y, x^{4}=z$ and $u_{(i, j, k, l)}^{1}=u_{t \ldots t x \ldots x y \ldots y z \ldots z}$ with $i$ times $t, j$ times $x, k$ times $y$, and $l$ times $z$.

The vector fields

$$
D_{x^{k}}=\frac{\partial}{\partial x^{k}}+\sum_{\# I \geq 0} \sum_{\alpha=1}^{m} u_{I+1_{k}}^{\alpha} \frac{\partial}{\partial u_{I}^{\alpha}}, \quad k \in\{1, \ldots, n\},
$$

$\left(i_{1}, \ldots, i_{k}, \ldots, i_{n}\right)+1_{k}=\left(i_{1}, \ldots, i_{k}+1, \ldots, i_{n}\right)$, are called total derivatives. They commute everywhere on $J^{\infty}(\pi):\left[D_{x^{i}}, D_{x^{j}}\right]=0$.

The evolutionary vector field associated to an arbitrary vector-valued smooth function $\varphi: J^{\infty}(\pi) \rightarrow \mathbb{R}^{m}$ is the vector field

$$
\mathbf{E}_{\varphi}=\sum_{\# I \geq 0} \sum_{\alpha=1}^{m} D_{I}\left(\varphi^{\alpha}\right) \frac{\partial}{\partial u_{I}^{\alpha}}
$$

with $D_{I}=D_{\left(i_{1}, \ldots i_{n}\right)}=D_{x^{1}}^{i_{1}} \circ \ldots \circ D_{x^{n}}^{i_{n}}$.

A system of PDEs $F_{r}\left(x^{i}, u_{I}^{\alpha}\right)=0$ of the order $s \geq 1$ with $\# I \leq s$, $r \in\{1, \ldots, R\}$ for some $R \geq 1$, defines the submanifold $\mathcal{E}=\left\{\left(x^{i}, u_{I}^{\alpha}\right) \in\right.$ $\left.J^{\infty}(\pi) \mid D_{K}\left(F_{r}\left(x^{i}, u_{I}^{\alpha}\right)\right)=0, \# K \geq 0\right\}$ in $J^{\infty}(\pi)$.

A function $\varphi: J^{\infty}(\pi) \rightarrow \mathbb{R}^{m}$ is called a (generator of an infinitesimal) symmetry of equation $\mathcal{E}$ when $\mathbf{E}_{\varphi}(F)=0$ on $\mathcal{E}$. The symmetry $\varphi$ is a solution to the defining system

$$
\ell_{\mathcal{E}}(\varphi)=0
$$


where $\ell_{\varepsilon}=\left.\ell_{F}\right|_{\varepsilon}$ with the matrix differential operator

$$
\ell_{F}=\left(\sum_{\# I \geq 0} \frac{\partial F_{r}}{\partial u_{I}^{\alpha}} D_{I}\right) .
$$

The symmetry algebra $\operatorname{Sym}(\mathcal{E})$ of equation $\mathcal{E}$ is the linear space of solutions to (11) endowed with the structure of a Lie algebra over $\mathbb{R}$ by the Jacobi bracket $\{\varphi, \psi\}=\mathbf{E}_{\varphi}(\psi)-\mathbf{E}_{\psi}(\varphi)$. The algebra of contact symmetries $\operatorname{Sym}_{0}(\mathcal{E})$ is the Lie subalgebra of $\operatorname{Sym}(\mathcal{E})$ defined as $\operatorname{Sym}(\mathcal{E}) \cap J^{1}(\pi)$.

Consider $\mathcal{W}=\mathbb{R}^{\infty}$ with coordinates $w^{s}, s \in \mathbb{N}_{0}$. Locally, an (infinitedimensional) differential covering of $\mathcal{E}$ is a trivial bundle $\tau: J^{\infty}(\pi) \times \mathcal{W} \rightarrow$ $J^{\infty}(\pi)$ equipped with extended total derivatives

$$
\widetilde{D}_{x^{k}}=D_{x^{k}}+\sum_{s=0}^{\infty} T_{k}^{s}\left(x^{i}, u_{I}^{\alpha}, w^{j}\right) \frac{\partial}{\partial w^{s}}
$$

such that $\left[\widetilde{D}_{x^{i}}, \widetilde{D}_{x^{j}}\right]=0$ for all $i \neq j$ whenever $\left(x^{i}, u_{I}^{\alpha}\right) \in \mathcal{E}$. Define the partial derivatives of $w^{s}$ by $w_{x^{k}}^{s}=\widetilde{D}_{x^{k}}\left(w^{s}\right)$. This yields the system of covering equations

$$
w_{x^{k}}^{s}=T_{k}^{s}\left(x^{i}, u_{I}^{\alpha}, w^{j}\right)
$$

that is compatible whenever $\left(x^{i}, u_{I}^{\alpha}\right) \in \mathcal{E}$.

Dually, the covering is defined by the Wahlquist-Estabrook forms

$$
d w^{s}-\sum_{k=1}^{m} T_{k}^{s}\left(x^{i}, u_{I}^{\alpha}, w^{j}\right) d x^{k}
$$

as follows: when $w^{s}$ and $u^{\alpha}$ are considered to be functions of $x^{1}, \ldots, x^{n}$, forms (13) are equal to zero whenever system (12) holds.

\subsection{Exotic cohomology of Lie algebras}

For a Lie algebra $\mathfrak{g}$ over $\mathbb{R}$, its representation $\rho: \mathfrak{g} \rightarrow \operatorname{End}(V)$, and $k \geq 1$ let $C^{k}(\mathfrak{g}, V)=\operatorname{Hom}\left(\Lambda^{k}(\mathfrak{g}), V\right)$ be the space of all $k$-linear skew-symmetric mappings from $\mathfrak{g}$ to $V$. Then the Chevalley-Eilenberg differential complex

$$
V=C^{0}(\mathfrak{g}, V) \stackrel{d}{\longrightarrow} C^{1}(\mathfrak{g}, V) \stackrel{d}{\longrightarrow} \ldots \stackrel{d}{\longrightarrow} C^{k}(\mathfrak{g}, V) \stackrel{d}{\longrightarrow} C^{k+1}(\mathfrak{g}, V) \stackrel{d}{\longrightarrow} \ldots
$$


is generated by the differential $d: \theta \mapsto d \theta$ such that

$$
\begin{aligned}
& d \theta\left(X_{1}, \ldots, X_{k+1}\right)=\sum_{q=1}^{k+1}(-1)^{q+1} \rho\left(X_{q}\right)\left(\theta\left(X_{1}, \ldots, \hat{X}_{q}, \ldots, X_{k+1}\right)\right) \\
& +\sum_{1 \leq p<q \leq k+1}(-1)^{p+q+1} \theta\left(\left[X_{p}, X_{q}\right], X_{1}, \ldots, \hat{X}_{p}, \ldots, \hat{X}_{q}, \ldots, X_{k+1}\right) .
\end{aligned}
$$

The cohomology groups of the complex $\left(C^{*}(\mathfrak{g}, V), d\right)$ are referred to as the cohomology groups of the Lie algebra $\mathfrak{g}$ with coefficients in the representation $\rho$. For the trivial representation $\rho_{0}: \mathfrak{g} \rightarrow \mathbb{R}, \rho_{0}: X \mapsto 0$, the cohomology groups are denoted by $H^{*}(\mathfrak{g})$.

Consider a Lie algebra $\mathfrak{g}$ over $\mathbb{R}$ with non-trivial first cohomology group $H^{1}(\mathfrak{g})$ and take a closed 1-form $\alpha$ on $\mathfrak{g}$ such that $[\alpha] \neq 0$. Then for any $c \in \mathbb{R}$ define new differential $d_{c \alpha}: C^{k}(\mathfrak{g}, \mathbb{R}) \rightarrow C^{k+1}(\mathfrak{g}, \mathbb{R})$ by the formula

$$
d_{c \alpha} \theta=d \theta-c \alpha \wedge \theta
$$

From $d \alpha=0$ it follows that $d_{c \alpha}^{2}=0$. The cohomology groups of the complex

$$
C^{1}(\mathfrak{g}, \mathbb{R}) \stackrel{d_{c \alpha}}{\longrightarrow} \ldots \stackrel{d_{c \alpha}}{\longrightarrow} C^{k}(\mathfrak{g}, \mathbb{R}) \stackrel{d_{c \alpha}}{\longrightarrow} C^{k+1}(\mathfrak{g}, \mathbb{R}) \stackrel{d_{c \alpha}}{\longrightarrow} \ldots
$$

are referred to as the exotic cohomology groups [36, 37] of $\mathfrak{g}$ and denoted by $H_{c \alpha}^{*}(\mathfrak{g})$.

\section{Hyper-CR equation}

\subsection{Contact symmetries}

Denote by $\varepsilon_{1}$ the hyper-CR equation (1). Direct computations 1 show that the Lie algebra $\operatorname{Sym}_{0}\left(\mathcal{E}_{1}\right)$ is generated by functions

$$
\begin{aligned}
& W_{0}(A)=-A u_{t}-\left(x A^{\prime}+\frac{1}{2} y^{2} A^{\prime \prime}\right) u_{x}-y A^{\prime} u_{y}+u A^{\prime}+x y A^{\prime \prime}+\frac{1}{6} y^{3} A^{\prime \prime \prime}, \\
& W_{1}(A)=-y A^{\prime} u_{x}-A u_{y}+x A^{\prime}+\frac{1}{2} y^{2} A^{\prime \prime} \\
& W_{2}(A)=-A u_{x}+y A^{\prime}
\end{aligned}
$$

\footnotetext{
${ }^{1}$ We carried out computations of generators of contact symmetries and their commutator tables in the Jets software [5].
} 


$$
\begin{aligned}
& W_{3}(A)=A, \\
& X_{0}=-2 x u_{x}-y u_{y}+3 u, \\
& X_{1}=-y u_{x}+2 x
\end{aligned}
$$

where $A=A(t)$ and $B=B(t)$ below are arbitrary functions of $t$. The commutators of the generators are given by equations

$$
\begin{cases}\left\{W_{i}(A), W_{j}(B)\right\} & =W_{i+j}\left(A B^{\prime}-B A^{\prime}\right) \\ \left\{X_{i}, W_{k}(A)\right\} & =-k W_{k+i}(A) \\ \left\{X_{0}, X_{1}\right\} & =-X_{1}\end{cases}
$$

where $W_{k}(A)=0$ for $k>3$. From equations (14) it follows that the contact symmetry algebra of $\mathcal{E}_{1}$ is the semi-direct product $\operatorname{Sym}_{0}\left(\mathcal{E}_{1}\right)=\mathfrak{p}_{4, \infty} \rtimes \mathfrak{p}_{\diamond}$ of the two-dimensional non-Abelian Lie algebra $\mathfrak{p}_{\diamond}=\left\langle X_{0}, X_{1}\right\rangle$ and the infinitedimensional ideal $\mathfrak{p}_{4, \infty}=\left\langle W_{k}(A) \mid 0 \leq k \leq 3\right\rangle$. The ideal, in its turn, is isomorphic to the tensor product $\mathbb{R}_{3}\left[h_{0}\right] \otimes \mathfrak{w}[t]$ of the four-dimensional commutative associative algebra of truncated polynomials $\mathbb{R}_{3}\left[h_{0}\right]=\mathbb{R}\left[h_{0}\right] /\left(h_{0}^{4}=0\right)$ and the Lie algebra $\mathfrak{w}[t]=\left\langle t^{i} \partial_{t} \mid i \in \mathbb{N}_{0}\right\rangle$.

Consider the basis of $\operatorname{Sym}_{0}\left(\mathcal{E}_{1}\right)$ given by generators $X_{0}, X_{1}$, and $\frac{1}{i !} W_{k}\left(t^{i}\right)$ with $i \in \mathbb{N}_{0}, k \in\{0, \ldots, 3\}$. Define the Maurer-Cartan forms $\alpha_{0}, \alpha_{1}, \theta_{k, i}$ for $\operatorname{Sym}_{0}\left(\mathcal{E}_{1}\right)$ as the dual forms to this basis: $\alpha_{m}\left(X_{j}\right)=\delta_{i j}, \alpha_{m}\left(W_{k}\left(t^{i}\right)\right)=0$, $\theta_{k, i}\left(X_{j}\right)=0, \theta_{k, i}\left(W_{k^{\prime}}\left(t^{i^{\prime}}\right)\right)=i ! \delta_{k k^{\prime}} \delta_{i i^{\prime}}$. Put

$$
\Theta=\sum_{k=0}^{3} \sum_{m=0}^{\infty} \frac{h_{0}^{k} h_{1}^{m}}{m !} \theta_{k, m}
$$

where $h_{0}$ and $h_{1}$ are formal parameters such that $d h_{i}=0$ and $h_{0}^{k}=0$ when $k>3$. Denote by $\nabla_{0}$ the derivative with respect to $h_{0}$ in $\mathbb{R}_{3}\left[h_{0}\right]$ and put $\nabla_{1}=\partial_{h_{1}}$. Then the commutator table (14) gives the Maurer-Cartan structure equations

$$
\left\{\begin{array}{l}
d \alpha_{0}=0 \\
d \alpha_{1}=\alpha_{0} \wedge \alpha_{1} \\
d \Theta=\nabla_{1}(\Theta) \wedge \Theta+\left(h_{0} \alpha_{0}+h_{0}^{2} \alpha_{1}\right) \wedge \nabla_{0}(\Theta)
\end{array}\right.
$$

of $\operatorname{Sym}_{0}\left(\mathcal{E}_{1}\right)$. 
REMARK. The last equation in system (16) is actually a short form of an infinite system that includes four series of equations for $\theta_{0, i}, \ldots, \theta_{3, i}$. For example, the first equations from each series are given as follows:

$$
\left\{\begin{aligned}
d \theta_{0,0}= & \theta_{0,1} \wedge \theta_{0,0}, \\
d \theta_{1,0}= & \left(\alpha_{0}+\theta_{0,1}\right) \wedge \theta_{1,0}+\theta_{1,1} \wedge \theta_{0,0}, \\
d \theta_{2,0}= & \left(2 \alpha_{0}+\theta_{0,1}\right) \wedge \theta_{2,0}+\left(\alpha_{1}+\theta_{1,1}\right) \wedge \theta_{1,0}+\theta_{2,1} \wedge \theta_{0,0}, \\
d \theta_{3,0}= & \left(3 \alpha_{0}+\theta_{0,1}\right) \wedge \theta_{3,0}+\left(2 \alpha_{1}+\theta_{1,1}\right) \wedge \theta_{2,0}+\theta_{2,1} \wedge \theta_{1,0} \\
& +\theta_{3,1} \wedge \theta_{0,0} .
\end{aligned}\right.
$$

Equations for all the other forms $\theta_{k, i}$ can be obtained from equations (17) by the procedure of normal prolongation, [8, 11]. For the purposes of the present paper we need explicit expressions for the first forms $\theta_{k, 0}$ of each series only. To shorten the notation we will write $\theta_{k}$ instead of $\theta_{k, 0}$ in this section or instead of $\theta_{k, 0,0}$ in the next sections.

\subsection{Second exotic cohomology group and non-central extension}

From the structure equations (16) we have

Proposition 1. $H^{1}\left(\operatorname{Sym}_{0}\left(\mathcal{E}_{1}\right)\right)=\left\langle\left[\alpha_{0}\right]\right\rangle=\left\langle\alpha_{0}\right\rangle$ and

$$
H_{c \alpha_{0}}^{2}\left(\mathfrak{p}_{\diamond}\right)= \begin{cases}\left\langle\left[\alpha_{0} \wedge \alpha_{1}\right]\right\rangle, & c=1, \\ \{[0]\}, & c \neq 1 .\end{cases}
$$

Furthermore, $H_{\alpha_{0}}^{2}\left(\mathfrak{p}_{\diamond}\right) \subseteq H_{\alpha_{0}}^{2}\left(\operatorname{Sym}_{0}\left(\mathcal{E}_{1}\right)\right)$. Hence the nontrivial 2-cocycle $\alpha_{0} \wedge$ $\alpha_{1}$ of the differential $d_{\alpha_{0}}$ defines a non-central extension $\widehat{\mathfrak{p}}_{\diamond}$ of the Lie algebra $\mathfrak{p}_{\diamond}$ and thus a non-central extension $\mathfrak{p}_{4, \infty} \rtimes \widehat{\mathfrak{p}}_{\diamond}$ of the Lie algbera $\operatorname{Sym}_{0}\left(\mathcal{E}_{1}\right)$. The additional Maurer-Cartan form $\sigma$ for the extended Lie algebra is a solution to $d_{\alpha_{0}} \sigma=\alpha_{0} \wedge \alpha_{1}$, that is, to equation

$$
d \sigma=\alpha_{0} \wedge \sigma+\alpha_{0} \wedge \alpha_{1} .
$$

This equation is compatible with the structure equations (16) of the Lie algebra $\operatorname{Sym}_{0}\left(\mathcal{E}_{1}\right)$.

\subsection{Maurer-Cartan forms and Lax representation}

We can compute the Maurer-Cartan forms $\alpha_{i}, \theta_{k}$, and $\sigma$ via two approaches. The first one is to integrate equations (16), (18) step by step. Each integration gives certain number of new coordinates (the 'integration 
constants') to express the new form, while it is not clear how these coordinates are related to the coordinates of $\mathcal{E}_{1}$. For example, from the first two equations of system (16) and equation (18) we obtain

$$
\alpha_{0}=d q, \quad \alpha_{1}=-\mathrm{e}^{q} d s, \quad \sigma=\mathrm{e}^{q}(d v-q d s),
$$

where $q, s$, and $v$ are free parameters 2 . The second approach to computing the Maurer-Cartan forms is to use Cartan's method of equivalence, [8, 9, 10, 11, 38, 16], see details and examples of applying the method to symmetries of PDEs in [29, 30]. For the symmetry algebra of equation (11) the combination of both techniques shows that

(i) $\theta_{0}$ is a multiple of $d t, \theta_{1}$ belongs to the algebraic ideal of 1 -forms generated by $d y, d t, \theta_{2}$ belongs to the ideal generated by $d x, d y, d t$;

(ii) $\theta_{3}$ is a multiple of the contact form $d u-u_{t} d t-u_{x} d x-u_{y} d y$.

Using (i) we have $\theta_{0}=r d t, \theta_{1}=\mathrm{e}^{q} r\left(d y+p_{1} d t\right), \theta_{2}=\mathrm{e}^{2 q} r\left(d x+\left(p_{1}-s\right) d y+\right.$ $\left.p_{2} d t\right)$, with new parameters $r \neq 0, p_{1}, p_{2}$, while (ii) then gives $p_{1}=-u_{x}+2 s$, $p_{2}=-u_{y}-s u_{x}+s^{2}$, and

$$
\theta_{3}=\mathrm{e}^{3 q} r\left(d u-u_{t} d t-u_{x} d x-u_{y} d y\right)
$$

Consider the linear combination

$$
\sigma-\theta_{2}=\mathrm{e}^{q}\left(d v-q d s-\mathrm{e}^{q} r\left(d x+\left(s^{2}-s u_{x}-u_{y}\right) d t+\left(s-u_{x}\right) d y\right)\right)
$$

and assume that $u$ and $v$ are functions of $t, x, y$. Then $\sigma-\theta_{2}=0$ implies $q=$ $v_{s}, r=v_{x} \exp \left(-v_{s}\right)$. After this change of notation we obtain the WahlquistEstabrook form

$$
\sigma-\theta_{2}=\mathrm{e}^{v_{s}}\left(d v-v_{s} d s-v_{x}\left(d x+\left(s^{2}-s u_{x}-u_{y}\right) d t+\left(s-u_{x}\right) d y\right)\right)
$$

of the Lax representation

$$
\left\{\begin{array}{l}
v_{t}=\left(s^{2}-s u_{x}-u_{y}\right) v_{x} \\
v_{y}=\left(s-u_{x}\right) v_{x}
\end{array}\right.
$$

This system differs from (7) by notation.

\footnotetext{
${ }^{2}$ we put $\alpha_{1}=-\mathrm{e}^{q} d s$ instead of the natural choice $\alpha_{1}=\mathrm{e}^{q} d s$ to simplify the computations below.
} 


\subsection{Integrable hierarchy associated to hyper-CR equation}

The Lie algebra $\mathfrak{p}_{4}=\mathfrak{p}_{4, \infty} \rtimes \mathfrak{p}_{\diamond}$ admits a sequence of natural extensions $\mathfrak{p}_{n+1}=\mathfrak{p}_{n+1, \infty} \rtimes \mathfrak{p}_{\diamond}, n \geq 4$, where $\mathfrak{p}_{n+1, \infty}=\mathbb{R}_{n}\left[h_{0}\right] \otimes \mathfrak{w}[t]$ and $\mathbb{R}_{n}\left[h_{0}\right]=$ $\mathbb{R}\left[h_{0}\right] /\left(h_{0}^{n+1}=0\right)$. These extensions are defined by the structure equations of the same form (16), where now we put

$$
\Theta=\sum_{k=0}^{n} \sum_{m=0}^{\infty} \frac{h_{0}^{k} h_{1}^{m}}{m !} \theta_{k, m}
$$

instead of (15) and assume $h_{0}^{k}=0$ for $k>n$. The finite-dimensional part $\mathfrak{p}_{\diamond}$ in all the algebras $\mathfrak{p}_{n+1}$ is the same, and for each $n \geq 4$ we have $H_{\alpha_{0}}^{2}\left(\mathfrak{p}_{\diamond}\right) \subseteq$ $H_{\alpha_{0}}^{2}\left(\mathfrak{p}_{n+1}\right)$. Hence the nontrivial 2-cocycle $\alpha_{0} \wedge \alpha_{1}$ of the differential $d_{\alpha_{0}}$ defines a non-central extension $\widehat{\mathfrak{p}}_{n+1}=\mathfrak{p}_{n+1, \infty} \rtimes \widehat{\mathfrak{p}}_{\diamond}$ of the Lie algebra $\mathfrak{p}_{n+1}$. The structure equations for $\widehat{\mathfrak{p}}_{n+1}$ are given by (16) , (20), and (18).

For a fixed $n \geq 4$ we can find forms $\theta_{k}$ with $0 \leq k \leq n$ by integration of the structure equations of $\mathfrak{p}_{n+1}$. In the next subsections we give examples of such computations. There we alter notation as follows: $t \mapsto t_{0}, y \mapsto t_{1}$, $x \mapsto t_{2}$.

\subsubsection{Case $n=4$.}

While the 1 -forms $\alpha_{0}, \alpha_{1}, \sigma, \theta_{0}$, and $\theta_{1}$ are the same as in section 3.3 , instead of (19) we have now

$$
\theta_{3}=\mathrm{e}^{3 q} r\left(d t_{3}+\left(p_{1}-2 s\right) d t_{2}+\left(p_{2}-s p_{1}+s^{2}\right) d t_{1}+p_{3} d t_{0}\right) .
$$

Next integration gives

$$
\begin{gathered}
\theta_{4}=\mathrm{e}^{4 q} r\left(d t_{4}+\left(p_{1}-3 s\right) d t_{3}+\left(p_{2}-2 s p_{1}+3 s^{2}\right) d t_{2}\right. \\
\left.+\left(p_{3}-s p_{2}+s^{2} p_{1}-s^{3}\right) d t_{1}+p_{4} d t_{0}\right) .
\end{gathered}
$$

We enforce $\theta_{4}$ to be the contact form $q^{4} r\left(d u-\sum_{i=0}^{3} u_{t_{i}} d t_{i}\right)$, that is, we put $t_{4}=u, p_{1}=-u_{t_{3}}+3 s, p_{2}=-u_{t_{2}}-2 s u_{t_{3}}+3 s^{2}, p_{3}=-u_{t_{1}}-s u_{t_{2}}+s^{2} u_{t_{3}}+s^{3}$, $p_{4}=-u_{t_{0}}$. Then we consider the linear combination

$$
\begin{aligned}
\sigma-\theta_{3}= & \mathrm{e}^{q}\left(d v-q d s-\mathrm{e}^{2 q} r\left(d t_{3}+\left(s-u_{t_{3}}\right) d t_{2}+\left(s^{2}-s u_{t_{3}}-u_{t_{2}}\right) d t_{1}\right.\right. \\
& \left.+\left(s^{3}-s^{2} u_{t_{3}}-s^{2} u_{t_{2}}-u_{t_{1}}\right) d t_{0}\right) .
\end{aligned}
$$


Substituting for $q=v_{s}, r=v_{t_{3}} \mathrm{e}^{-v_{s}}$ yields

$$
\begin{aligned}
\sigma-\theta_{0}= & \mathrm{e}^{v_{s}}\left(d v-v_{s} d s-v_{t_{3}}\left(d t_{3}+\left(s-u_{t_{3}}\right) d t_{2}+\left(s^{2}-s u_{t_{3}}-u_{t_{2}}\right) d t_{1}\right.\right. \\
& \left.+\left(s^{3}-s^{2} u_{t_{3}}-s^{2} u_{t_{2}}-u_{t_{1}}\right) d t_{0}\right) .
\end{aligned}
$$

We consider $u$ as a function of $t_{0}, \ldots, t_{3}$ and $v$ as a function of $t_{0}, \ldots, t_{3}$, $s$. Then equation (21) defines the Wahlquist-Estabrook form for the Lax representation

$$
\left\{\begin{array}{l}
v_{t_{2}}=\left(s-u_{t_{3}}\right) v_{t_{3}}, \\
v_{t_{1}}=\left(s^{2}-s u_{t_{3}}-u_{t_{2}}\right) v_{t_{3}}, \\
v_{t_{0}}=\left(s^{3}-s^{2} u_{t_{3}}-s^{2} u_{t_{2}}-u_{t_{1}}\right) v_{t_{3}}
\end{array}\right.
$$

of a system of PDEs.

To make the structures of this Lax representation and of the defined system more tractable we alter notation by substituting $t_{i} \mapsto x_{3-i}$ for $i \in$ $\{0, \ldots, 3\}$. The obtained system

$$
\left\{\begin{array}{l}
v_{x_{1}}=\left(s-u_{x_{0}}\right) v_{x_{0}} \\
v_{x_{2}}=\left(s^{2}-s u_{x_{0}}-u_{x_{1}}\right) v_{x_{0}} \\
v_{x_{3}}=\left(s^{3}-s^{2} u_{x_{0}}-s^{2} u_{x_{1}}-u_{x_{2}}\right) v_{x_{0}}
\end{array}\right.
$$

is compatible whenever there holds

$$
\begin{aligned}
& u_{x_{1} x_{1}}=u_{x_{0} x_{2}}+u_{x_{1}} u_{x_{0} x_{0}}-u_{x_{0}} u_{x_{0} x_{1}}, \\
& u_{x_{1} x_{2}}=u_{x_{0} x_{3}}+u_{x_{2}} u_{x_{0} x_{0}}-u_{x_{0}} u_{x_{0} x_{2}}, \\
& u_{x_{1} x_{3}}=u_{x_{2} x_{2}}+u_{x_{1}} u_{x_{0} x_{2}}-u_{x_{2}} u_{x_{0} x_{1}} .
\end{aligned}
$$

Equations (22), (23), (24) differ from equations (11), (22), (3), respectively, by notation.

\subsubsection{Case $n>4$.}

For the Lie algebra $\widehat{\mathfrak{p}}_{n+1}$ with fixed $n>4$ the results of computations are the following. Put $p_{0}=1$ and for $i \geq 0, j \in\{0, \ldots, i\}$ define polynomials $P_{i j}=P_{i j}(s)$ of variable $s$ by the formula

$$
P_{i j}=\sum_{k=0}^{j}(-1)^{k}\left(\begin{array}{c}
i-j+k-1 \\
k
\end{array}\right) p_{j-k} s^{k} .
$$


Coefficients of $P_{i j}$ depend on parameters $p_{1}, \ldots, p_{j}$. Then we have expressions

$$
\theta_{k}=\mathrm{e}^{k q} r \sum_{j=0}^{k} P_{k j} d t_{k-j}
$$

for forms $\theta_{k}$ with $k \in\{0, \ldots, n\}$. We put $t_{n}=u$, solve the triangular linear system of equations

$$
P_{n, n-i}=-u_{t_{i}}, \quad i \in\{0, \ldots, n-1\}
$$

with respect to unknowns $p_{1}, p_{2}, \ldots, p_{n}$, and then alter notation by substituting $t_{i}=x_{n-i}$ for $i \in\{0, \ldots, n-1\}$. This yields

$$
\theta_{n}=\mathrm{e}^{n q} r\left(d u-\sum_{i=0}^{n-1} u_{x_{i}} d x_{i}\right) .
$$

Then we consider the linear combination $\sigma-\theta_{n-1}$ and put $q=v_{s}, r=$ $v_{x_{0}} \mathrm{e}^{(1-n) v_{s}}$. This produces the Wahlquist-Estabrook form

$$
\sigma-\theta_{n-1}=\mathrm{e}^{v_{s}}\left(d v-v_{s} d s-v_{x_{0}} d x_{0}-\sum_{i=1}^{n-1}\left(s^{i}-\sum_{j=0}^{i-1} s^{i-j-1} u_{x_{j}}\right) v_{x_{0}} d x_{i}\right)
$$

for the Lax representation

$$
\left\{\begin{aligned}
v_{x_{1}}= & \left(s-u_{x_{0}}\right) v_{x_{0}}, \\
v_{x_{2}}= & \left(s^{2}-s u_{x_{0}}-u_{x_{1}}\right) v_{x_{0}}, \\
& \cdots \\
v_{x_{i}}= & \left(s^{i}-\sum_{j=0}^{i-1} s^{i-j-1} u_{x_{j}}\right) v_{x_{0}}, \\
& \cdots \\
v_{x_{n-1}}= & \left(s^{n-1}-s^{n-2} u_{x_{0}}-s^{n-3} u_{x_{1}}-\ldots-s u_{x_{n-3}}-u_{x_{n-2}}\right) v_{x_{0}} .
\end{aligned}\right.
$$

Denote by $\mathcal{H}_{n-1}$ the compatibility conditions for system (26). Then $\mathcal{H}_{2}$ is given by the single equation (22), this equation supplemented by equations (23), (24) defines $\mathcal{H}_{3}$, system $\mathcal{H}_{4}$ consists of equations from $\mathcal{H}_{3}$ supplemented by equations

$$
\begin{aligned}
& u_{x_{0} x_{4}}=u_{x_{2} x_{2}}+u_{x_{0}} u_{x_{0} x_{3}}-u_{x_{3}} u_{x_{0} x_{0}}+u_{x_{1}} u_{x_{0} x_{2}}-u_{x_{2}} u_{x_{0} x_{1}}, \\
& u_{x_{1} x_{4}}=u_{x_{2} x_{3}}+u_{x_{0}} u_{x_{0} x_{3}}-u_{x_{3}} u_{x_{0} x_{1}},
\end{aligned}
$$




$$
u_{x_{2} x_{4}}=u_{x_{3} x_{3}}+u_{x_{2}} u_{x_{0} x_{3}}-u_{x_{3}} u_{x_{0} x_{2}}
$$

etc., system $\mathcal{H}_{n-1}$ consists of equations from $\mathcal{H}_{n-2}$ supplemented by equations

$$
u_{x_{i-1} x_{n}}=u_{x_{i} x_{n-1}}+u_{x_{0}} u_{x_{0} x_{n-1}}-u_{x_{n-1}} u_{x_{0} x_{i-1}}, \quad i \in\{1, \ldots, n-2\},
$$

where $u_{x_{i} x_{n-1}}$ are replaced by the right-hand sides of the equations from $\mathcal{H}_{n-2}$. The Lax representations (26) and systems $\mathcal{H}_{n}$ were introduced in [15], see also [39, 7].

\section{Reduced quasi-classical self-dual Yang-Mills equation}

Equation (22) differs by notation from equation (34) in the hierarchies $\mathcal{H}_{m}$ with $m \geq 3$. While the symmetry algebra $\operatorname{Sym}_{0}\left(\varepsilon_{2}\right)$ of equation (2) has more complicated structure than $\operatorname{Sym}_{0}\left(\mathcal{E}_{1}\right)$, we show that the Lax representation (81) as well as the integrable hierarchy associated to (2) can be inferred from the Maurer-Cartan forms of the non-central extension generated by the nontrivial exotic 2-cocycle of $\operatorname{Sym}_{0}\left(\mathcal{E}_{2}\right)$.

\subsection{Contact symmetries}

The Lie algebra $\operatorname{Sym}_{0}\left(\mathcal{E}_{2}\right)$ admits generators

$$
\begin{aligned}
W_{0}(A) & =-\left(A_{z} x+A_{t} y\right) u_{x}-A u_{z}+A_{z} u+\frac{1}{2} A_{z z} x^{2}+A_{t z} x y,+\frac{1}{2} A_{t t} y^{2}, \\
W_{1}(A) & =-A u_{x}+A_{z} x+A_{t} y \\
W_{2}(A) & =A \\
X & =-x u_{x}-y u_{y}+2 u \\
Y_{0} & =-u_{t} \\
Y_{1} & =-t u_{t}+\frac{1}{2}\left(x u_{x}-y u_{y}\right)-u, \\
Y_{2} & =-\frac{1}{2}\left(t^{2} u_{t}+t x u_{x}-t y u_{y}-x y\right)-t u \\
Z_{0} & =-u_{y} \\
Z_{1} & =-t u_{y}-x
\end{aligned}
$$

where $A=A(t, z)$ are arbitrary functions. The commutator table of $\operatorname{Sym}_{0}\left(\varepsilon_{2}\right)$ is given by equations

$$
\begin{aligned}
& {\left[W_{i}(A), W_{j}(B)\right]= \begin{cases}W_{i+j}\left(A B_{z}-B A_{z}\right), & i+j \leq 2 \\
0, & i+j>2,\end{cases} } \\
& {\left[X, W_{k}(A)\right]=-k W_{k}(A),}
\end{aligned}
$$




$$
\begin{aligned}
& {\left[Y_{0}, W_{k}(A)\right]=W_{k}\left(A_{t}\right)} \\
& {\left[Y_{1}, W_{k}(A)\right]=W_{k}\left(t A_{t}+\frac{1}{2} k A\right),} \\
& {\left[Y_{2}, W_{k}(A)\right]=\frac{1}{2} W_{k}\left(t^{2} A_{t}+k t A\right),} \\
& {\left[Z_{0}, W_{k}(A)\right]= \begin{cases}W_{k+1}\left(A_{t}\right), & k \leq 1 \\
0, & k=2\end{cases} } \\
& {\left[Z_{1}, W_{k}(A)\right]= \begin{cases}W_{k+1}\left(t A_{t}+k A\right), & k \leq 1 \\
0, & k=2\end{cases} } \\
& {\left[X, Y_{m}\right]=0, \quad\left[X, Z_{m}\right]=-Z_{m}, \quad\left[Z_{0}, Z_{1}\right]=0,} \\
& {\left[Y_{0}, Y_{1}\right]=Y_{0}, \quad\left[Y_{0}, Y_{2}\right]=Y_{1}, \quad\left[Y_{1}, Y_{2}\right]=Y_{2},} \\
& {\left[Y_{0}, Z_{0}\right]=0, \quad\left[Y_{1}, Z_{0}\right]=-\frac{1}{2} Z_{0}, \quad\left[Y_{2}, Z_{0}\right]=-\frac{1}{2} Z_{1} \text {, }} \\
& {\left[Y_{0}, Z_{1}\right]=Z_{0}, \quad\left[Y_{1}, Z_{1}\right]=\frac{1}{2} Z_{1}, \quad\left[Y_{2}, Z_{1}\right]=0 \text {. }}
\end{aligned}
$$

From this table it follows that $\operatorname{Sym}_{0}\left(\mathcal{E}_{2}\right)$ is the semi-direct product $\mathfrak{q}_{3}=$ $\mathfrak{q}_{3, \infty} \rtimes \mathfrak{q}_{\diamond}$ of the finite-dimensional Lie algebra $\mathfrak{q}_{\diamond}$ generated by $X, Y_{i}, Z_{j}$ and the infinite-dimensional ideal $\mathfrak{q}_{3, \infty}$ generated by $W_{0}(A), W_{1}(A), W_{2}(A)$. We have $\mathfrak{q}_{\diamond}=\mathfrak{a} \ltimes\left(\mathfrak{s l}_{2}(\mathbb{R}) \ltimes \mathfrak{v}\right)$, where $\mathfrak{a}=\langle X\rangle$ is one-dimensional Lie algebra, $\mathfrak{s l}_{2}(\mathbb{R})=\left\langle Y_{0}, Y_{1}, Y_{2}\right\rangle$, and $\mathfrak{v}=\left\langle Z_{0}, Z_{1}\right\rangle$ is two-dimensional Abelian Lie algebra, while $\mathfrak{q}_{3, \infty}$ is isomorphic to the tensor product $\mathbb{R}_{2}\left[h_{0}\right] \otimes \mathfrak{w}[t, z]$, where we denote $\mathfrak{w}[t, z]=\left\langle t^{i} z^{j} \partial_{z} \mid i, j \in \mathbb{N}_{0}\right\rangle$.

\subsection{Maurer-Cartan forms and the second exotic cohomology group}

Consider the Maurer-Cartan forms $\alpha, \beta_{i}, i \in\{0,1,2\}, \gamma_{l}, l \in\{0,1\}$, $\theta_{k, i, j}, k \in\{0,1,2\}, i, j \in \mathbb{N}_{0}$, of the Lie algebra $\mathfrak{q}_{3}$ that are dual to the basis $X, Y_{i}, Z_{l}, \frac{1}{i !} \frac{1}{j !} W_{k}\left(t^{i} z^{j}\right)$, in other words, take 1-forms such that there hold $\alpha(X)=1, \beta_{i}\left(Y_{i^{\prime}}\right)=\delta_{i i^{\prime}}, \gamma_{l}\left(Z_{l^{\prime}}\right)=\delta_{l l^{\prime}}, \theta_{k, i, j}\left(W_{k^{\prime}}\left(t^{i^{\prime}} z^{j^{\prime}}\right)\right)=i ! j ! \delta_{k k^{\prime}} \delta_{i i^{\prime}} \delta_{j j^{\prime}}$, while all the other values of these 1-forms on the elements of the basis are equal to zero. Denote

$$
B=\beta_{0}+h_{1} \beta_{1}+\frac{1}{2} h_{1}^{2} \beta_{2}, \quad \Gamma=\gamma_{0}+h_{1} \gamma_{1},
$$

and consider the formal series of 1 -forms

$$
\Theta=\sum_{k=0}^{2} \sum_{i=0}^{\infty} \sum_{j=0}^{\infty} \frac{h_{0}^{k} h_{1}^{i} h_{2}^{j}}{i ! j !} \theta_{k, i, j},
$$


Then the commutator table for the generators of $\mathfrak{q}_{3}$ yields the structure equations

$$
\left\{\begin{aligned}
d \alpha= & 0 \\
d B= & \nabla_{1}(B) \wedge B \\
d \Gamma= & \alpha \wedge \Gamma+\nabla_{1}(\Gamma) \wedge B+\frac{1}{2} \nabla_{1}(B) \wedge \Gamma \\
d \Theta= & \nabla_{2}(\Theta) \wedge \Theta+h_{0} \nabla_{0}(\Theta) \wedge\left(\frac{1}{2} \nabla_{1}(B)+h_{0} \nabla_{1}(\Gamma)-\alpha\right) \\
& +\nabla_{1}(\Theta) \wedge\left(B+h_{0} \Gamma\right) .
\end{aligned}\right.
$$

This system implies the following statement.

Proposition 2. $H^{1}\left(\mathfrak{q}_{3}\right)=\langle\alpha\rangle$,

$$
H_{c \alpha}^{2}\left(\mathfrak{q}_{\diamond}\right)= \begin{cases}\left\langle\left[\gamma_{0} \wedge \gamma_{1}\right]\right\rangle, & c=2, \\ \{[0]\}, & c \neq 2,\end{cases}
$$

and $H_{2 \alpha}^{2}\left(\mathfrak{q}_{\diamond}\right) \subseteq H_{2 \alpha}^{2}\left(\mathfrak{q}_{3}\right)$. Equation

$$
d \sigma=2 \alpha \wedge \sigma+\gamma_{0} \wedge \gamma_{1}
$$

with unknown 1-form $\sigma$ is compatible with the structure equations (28). System (28), (29) defines the structure equations for a non-central extension $\widehat{\mathfrak{q}}_{3}$ of the Lie algebra $\mathfrak{q}_{3}$.

\subsection{Lax representation of rqsdYM}

Integration of the structure equations (28), (29) gives consequently

$$
\begin{aligned}
& \alpha=\frac{d a_{0}}{a_{0}}, \quad \beta_{0}=a_{1}^{2} d t, \quad \beta_{1}=2 \frac{d a_{1}}{a_{1}}+a_{2} d t, \quad \beta_{2}=\frac{1}{a_{1}^{2}}\left(d a_{2}+\frac{a_{2}^{2}}{2} d t\right) \\
& \gamma_{0}=a_{0} a_{1}(d y+s d t), \quad \gamma_{1}=\frac{a_{0}}{a_{1}}\left(d s+\frac{1}{2} a_{2}(d y+s d t)\right) \\
& \sigma=a_{0}^{2}\left(d v-s d y-\frac{1}{2} s^{2} d t\right), \quad \theta_{0}=b\left(d z+q_{0} d t\right) \\
& \theta_{1}=\frac{a_{0} b}{a_{1}}\left(d x_{1}+p_{1} d z+q_{0} d y+q_{1} d t\right) \\
& \theta_{2}=\frac{a_{0}^{2} b}{a_{1}^{2}}\left(d x_{2}+\left(p_{1}-s\right) d x_{1}+p_{0} d z+\left(q_{1}-s q_{0}\right) d y+q_{2} d t\right)
\end{aligned}
$$


where $a_{0} \neq 0, a_{1} \neq 0$, and $b \neq 0$. We put $x_{2}=u, p_{1}=-u_{x}+s, p_{2}=-u_{z}$, $q_{1}=-u_{y}+s q_{0}, q_{2}=-u_{t}$. This yields

$$
\theta_{2}=\frac{a_{0}^{2} b}{a_{1}^{2}}\left(d u-u_{t} d t-u_{x} d x-u_{y} d y-u_{z} d z\right)
$$

Then we alter notation by substituting $b=v_{x} v_{s}^{-1}, a_{1}=a_{0}^{-1} v_{s}^{-1}, q_{0}=v_{x}^{-1}\left(v_{y}-\right.$ $\left.\frac{1}{2} a_{2} v_{s}-s\right)$ in the linear combination

$$
\begin{aligned}
\mu_{1}=\sigma- & \gamma_{1}-\theta_{1}=a_{0}^{2}\left(d u-a_{0}^{-1} a_{1}^{-1}\left(d s+b\left(d x+\left(s-u_{x}\right) d z\right)\right.\right. \\
& +\left(b q_{0}+a_{0} a_{1} s+\frac{1}{2} a_{2}\right) d y+\left(b\left(q_{0} s-u_{y}\right)+\frac{1}{2} s\left(a_{2} s+a_{0} a_{1}\right) d t\right)
\end{aligned}
$$

and obtain

$\mu_{1}=a_{0}^{2}\left(d u-v_{s} d s-v_{x}\left(d x+\left(s-u_{x}\right) d z\right)-v_{y} d y-\left(s v_{y}-u_{y} v_{x}-\frac{1}{2} s^{2}\right) d t\right)$.

This is the Wahlquist-Estabrook form for the Lax representation

$$
\left\{\begin{array}{l}
v_{z}=\left(s-u_{x}\right) v_{x} \\
v_{t}=s v_{y}-u_{y} v_{x}-\frac{1}{2} s^{2}
\end{array}\right.
$$

of equation (21). System (31) differs from (8) by the change of notation $s \mapsto \lambda$, $v \mapsto v-\frac{1}{2} \lambda^{2} t$.

\subsection{Integrable hierarchy associated to rqsd $Y M$}

The Lie algebra $\mathfrak{q}_{3}$ admits a series of natural extensions $\mathfrak{q}_{n+1}=\mathfrak{q}_{n+1, \infty} \rtimes \mathfrak{q}_{\diamond}$ for $n \geq 3$ with $\mathfrak{q}_{n+1, \infty}=\mathbb{R}_{n+1}\left[h_{0}\right] \otimes \mathfrak{w}[t, z]$. The structure equations for the Lie algebra $\mathfrak{q}_{n+1}$ are given by system (28), (29), where we put

$$
\Theta=\sum_{k=0}^{n} \sum_{i=0}^{\infty} \sum_{j=0}^{\infty} \frac{h_{0}^{k} h_{1}^{i} h_{2}^{j}}{i ! j !} \theta_{k, i, j}
$$

instead of (27) and assume $h_{0}^{k}=0$ for $k>n$. The finite-dimensional part $\mathfrak{q}_{\diamond}$ in all the algebras $\mathfrak{q}_{n+1}$ is the same, and we have $H_{2 \alpha_{0}}^{2}\left(\mathfrak{q}_{\diamond}\right) \subseteq H_{2 \alpha_{0}}^{2}\left(\mathfrak{q}_{n+1}\right)$ for each $n \geq 3$. Hence the nontrivial 2-cocycle $\gamma_{0} \wedge \gamma_{1}$ of the differential $d_{2 \alpha_{0}}$ defines a non-central extension $\widehat{\mathfrak{q}}_{n+1}=\mathfrak{q}_{n+1, \infty} \rtimes \widehat{\mathfrak{q}}_{\diamond}$ of the Lie algbera $\mathfrak{q}_{n+1}$. The structure equations for $\widehat{\mathfrak{q}}_{n+1}$ are given by system (28), (29) with $\Theta$ defined by (32). To unify notation we rename $t \mapsto y_{1}, x \mapsto t_{0}, y \mapsto y_{0}$, $z \mapsto t_{1}$. Integration of the structure equations for a fixed $n \geq 4$ gives

$$
\theta_{k}=\frac{a_{0}^{k} b}{a_{1}^{k}}\left(\sum_{i=0}^{k} P_{k i} d t_{k-i}+\left(\sum_{i=0}^{k-1}(-1)^{i} q_{n-1-i} s^{i}\right) d y_{0}+q_{k} d y_{1}\right)
$$


where $k \in\{3, \ldots, n\}$, polynomials $P_{k i}$ are defined by (25) $, t_{0}, \ldots, t_{n}, q_{0}, \ldots$, $q_{n}$ are parameters, and forms $\alpha, \beta_{i}, \gamma_{j}, \sigma, \theta_{0}, \ldots, \theta_{2}$ are given by system (30). We put $t_{n}=u$ and rename $t_{i}=x_{n-1-i}, i \in\{0, \ldots, n-1\}$ to simplify notation in what follows. Then we solve the triangular linear system of equations $P_{n i}=-u_{x_{i}}, i \in\{0, \ldots, n-1\}$, with respect to $p_{1}, \ldots, p_{n}$ and put $q_{n}=-u_{y_{1}}, q_{n-1}=-u_{y_{0}}-\sum_{i=1}^{n-1}(-1)^{i} q_{n-1-i} s^{i}$. This yields the contact form

$$
\theta_{n}=\frac{a_{0}^{k} b}{a_{1}^{k}}\left(d u-u_{y_{0}} d y_{0}-u_{y_{1}} d y_{1}-\sum_{i=0}^{n-1} u_{x_{i}} d x_{i}\right)
$$

Then we consider the linear combination $\mu_{n}=\sigma-\gamma_{1}-\theta_{n-1}$ and put $b=$ $a_{0}^{-n-1} v_{x_{0}} v_{s}^{1-n}, a_{1}=a_{0}^{-1} v_{s}^{-1}, q_{n-2}=\left(v_{y_{0}}-s-\frac{1}{2} a_{2} v_{s}\right) v_{x_{0}}^{-1}+\sum_{i=1}^{n-2}(-1)^{i} q_{n-1-i} s^{i}$.

After this change of notation we obtain

$$
\begin{aligned}
\mu_{n}=a_{0}^{2} & \left(d v-v_{y_{0}} d y_{0}-\left(s v_{y_{0}}-u_{y_{0}} v_{x_{0}}-\frac{1}{2} s^{2}\right) d y_{1}-v_{s} d s-v_{x_{0}} d x_{0}\right. \\
& \left.-\sum_{i=1}^{n-1}\left(s^{i}-\sum_{j=0}^{i-1} s^{i-j-1} u_{x_{j}}\right) v_{x_{0}} d x_{i}\right) .
\end{aligned}
$$

This Wahlquist-Estabrook form defines the Lax representation

$$
\left\{\begin{aligned}
v_{y_{1}}= & s v_{y_{0}}-u_{y_{0}} v_{x_{0}}-\frac{1}{2} s^{2} \\
v_{x_{1}}= & \left(s-u_{x_{0}}\right) v_{x_{0}} \\
v_{x_{2}}= & \left(s^{2}-s u_{x_{0}}-u_{x_{1}}\right) v_{x_{0}} \\
& \cdots \\
v_{x_{i}}= & \left(s^{i}-\sum_{j=0}^{i-1} s^{i-j-1} u_{x_{j}}\right) v_{x_{0}} \\
& \cdots \\
v_{x_{n-1}}= & \left(s^{n-1}-s^{n-2} u_{x_{0}}-s^{n-3} u_{x_{1}}-\ldots-s u_{x_{n-3}}-u_{x_{n-2}}\right) v_{x_{0}} .
\end{aligned}\right.
$$

Equations for $v_{x_{i}}$ coincide with system (26). The compatibility conditions of system (33) define the integrable hierarchy associated to equation (2). This hierarchy includes system $\mathcal{H}_{n-1}$, equation (2) written as

$$
u_{x_{0} y_{1}}=u_{x_{1} y_{0}}-u_{y_{0}} u_{x_{0} x_{0}}-u_{x_{0}} u_{x_{0} y_{0}}
$$

and system

$$
u_{x_{i} y_{1}}=u_{x_{i+1} y_{0}}+u_{x_{i}} u_{x_{0} y_{0}}-u_{y_{0}} u_{x_{0} x_{i}}, \quad i \in\{0, \ldots, n-2\} .
$$




\section{The four-dimensional universal hierarchy equation}

In this section we consider equation $\mathcal{E}_{3}$ defined by (3). This equation differs by notation from (24). We show that the Lax representation (9) for equation (3) can be revealed independently from the hierarchies $\mathcal{H}_{n}$ in the previous sections by applying twice the procedure of non-central extension via notrivial exotic 2-cocycles to the symmetry algebra $\operatorname{Sym}_{0}\left(\mathcal{E}_{3}\right)$. Furthermore, we find an independent hierarchy associated to $\varepsilon_{3}$.

\subsection{Contact symmetries}

The Lie algebra $\operatorname{Sym}_{0}\left(\varepsilon_{3}\right)$ has generators

$$
\begin{aligned}
W_{0}(A) & =-A u_{y}+A_{y} u-A_{t} z, & W_{1}(A) & =A, \\
X_{1} & =-t u_{t}+x u_{x}-u, & X_{2} & =-u_{t}, \\
X_{3} & =-2 x u_{x}-z u_{z}+u, & X_{4} & =-\frac{1}{2} z u_{x}-t u_{z}, \\
X_{5} & =-u_{z}, & X_{6} & =-u_{x},
\end{aligned}
$$

where $A=A(t, y)$ and $B=B(t, y)$ below are arbitrary functions, and the commutator table

$$
\begin{aligned}
& \left\{W_{i}(A), W_{j}(B)\right\}=W_{i+j}\left(A B_{y}-B A_{y}\right), \\
& \left\{X_{1}, W_{0}(A)\right\}=W_{0}\left(t A_{t}\right), \quad\left\{X_{1}, W_{1}(A)\right\}=W_{1}\left(t A_{t}\right), \\
& \left\{X_{2}, W_{0}(A)\right\}=W_{0}\left(A_{t}\right), \quad\left\{X_{2}, W_{1}(A)\right\}=W_{1}\left(A_{t}\right), \\
& \left\{X_{3}, W_{0}(A)\right\}=0, \quad\left\{X_{3}, W_{1}(A)\right\}=-W_{1}(A), \\
& \left\{X_{4}, W_{0}(A)\right\}=-W_{1}\left(t A_{t}\right), \quad\left\{X_{4}, W_{1}(A)\right\}=0, \\
& \left\{X_{5}, W_{0}(A)\right\}=-W_{1}\left(A_{t}\right), \quad\left\{X_{5}, W_{1}(A)\right\}=0, \\
& \left\{X_{6}, W_{0}(A)\right\}=0, \quad\left\{X_{6}, W_{1}(A)\right\}=0, \\
& \left\{X_{1}, X_{2}\right\}=-X_{2}, \quad\left\{X_{1}, X_{4}\right\}=X_{4} \\
& \left\{X_{1}, X_{6}\right\}=X_{6}, \quad\left\{X_{2}, X_{4}\right\}=X_{5} \\
& \left\{X_{3}, X_{4}\right\}=-X_{4}, \quad\left\{X_{3}, X_{5}\right\}=-X_{5}, \\
& \left\{X_{3}, X_{6}\right\}=-2 X_{6}, \quad\left\{X_{4}, X_{5}\right\}=-\frac{1}{2} X_{5},
\end{aligned}
$$

while $\left\{X_{i}, X_{j}\right\}=0$ for all the other pairs $i<j$. The table shows that $\operatorname{Sym}_{0}\left(\mathcal{E}_{3}\right)=\mathfrak{r}_{2, \infty} \rtimes \mathfrak{r}_{\diamond}$, where $\mathfrak{r}_{\diamond}=\left\langle X_{m} \mid m \in\{1, \ldots, 6\}\right\rangle$ and $\mathfrak{r}_{2, \infty}=\mathbb{R}_{1}\left[h_{0}\right] \otimes$ $\mathfrak{w}[t, y]$.

Define the Maurer-Cartan forms $\theta_{k, i, j}$ with $k \in\{0,1\}, i, j \in \mathbb{N}_{0}$, and $\beta_{m}$ with $m \in\{1, \ldots, 6\}$, for the Lie algebra $\operatorname{Sym}_{0}\left(\mathcal{E}_{3}\right)$ as dual 1-forms to 
its basis $\frac{1}{i !} \frac{1}{j !} W_{k}\left(t^{i} y^{j}\right), X_{m}$, that is, put $\theta_{k, i, j}\left(W_{k^{\prime}}\left(t^{i^{\prime}} y^{j^{\prime}}\right)\right)=i ! j ! \delta_{k k^{\prime}} \delta_{i i^{\prime}} \delta_{j j^{\prime}}$, $\theta_{k, i, j}\left(X_{m}\right)=0, \beta_{m}\left(W_{k}\left(t^{i} y^{j}\right)\right)=0, \beta_{m}\left(X_{m^{\prime}}\right)=\delta_{m m^{\prime}}$. Denote

$$
\Theta_{k}=\sum_{i=0}^{\infty} \sum_{j=0}^{\infty} \frac{h_{1}^{i}}{i !} \frac{h_{2}^{j}}{j !} \theta_{k, i, j}
$$

then the system of the Maurer-Cartan structure equations for $\operatorname{Sym}_{0}\left(\mathcal{E}_{3}\right)$ is the union of systems

$$
\left\{\begin{array}{l}
d \beta_{1}=0 \\
d \beta_{2}=\beta_{1} \wedge \beta_{2} \\
d \beta_{3}=0 \\
d \beta_{4}=\left(\beta_{3}-\beta_{1}\right) \wedge \beta_{4} \\
d \beta_{5}=\beta_{3} \wedge \beta_{5}-\beta_{2} \wedge \beta_{4} \\
d \beta_{6}=\left(2 \beta_{3}-\beta_{1}\right) \wedge \beta_{6}+\frac{1}{2} \beta_{4} \wedge \beta_{5}
\end{array}\right.
$$

and

$$
\left\{\begin{aligned}
d \Theta_{0}=\nabla_{2}\left(\Theta_{0}\right) & \wedge \Theta_{0}+\nabla_{1}\left(\Theta_{0}\right) \wedge\left(\beta_{2}+h_{1} \beta_{1}\right) \\
d \Theta_{1}=\nabla_{2}\left(\Theta_{1}\right) & \wedge \Theta_{0}+\nabla_{2}\left(\Theta_{0}\right) \wedge \Theta_{1}+\left(\beta_{1}-\beta_{3}\right) \wedge \Theta_{1} \\
& +\left(\beta_{2}+h_{1} \beta_{1}\right) \wedge \nabla_{1}\left(\Theta_{1}\right)+\left(\beta_{5}+h_{1} \beta_{4}\right) \wedge \nabla_{1}\left(\Theta_{0}\right)
\end{aligned}\right.
$$

where (36) is the system of the structure equations for $\mathfrak{r}_{\diamond}$.

\subsection{Non-central extensions, Maurer-Cartan forms and Lax representation}

Direct computations using the structure equations (36), (37) give the following statement.

Propositon 3. $H^{1}\left(\operatorname{Sym}\left(\mathcal{E}_{3}\right)\right)=\left\langle\beta_{1}, \beta_{3}\right\rangle$ and

$$
H_{c_{1} \beta_{1}+c_{2} \beta_{3}}^{2}\left(\mathfrak{r}_{\diamond}\right)=\left\{\begin{array}{lll}
\left\langle\left[\beta_{2} \wedge \beta_{5}\right]\right\rangle, & c_{1}=1, & c_{2}=1, \\
\left\langle\left[\beta_{1} \wedge \beta_{2}\right],\left[\beta_{2} \wedge \beta_{3}\right]\right\rangle, & c_{1}=1, & c_{2}=0, \\
\left\langle\left[\beta_{1} \wedge \beta_{4}\right],\left[\beta_{3} \wedge \beta_{4}\right]\right\rangle, & c_{1}=-1, & c_{2}=1, \\
\left\langle\left[\beta_{4} \wedge \beta_{6}\right]\right\rangle, & c_{1}=-2, & c_{2}=3, \\
\{[0]\}, & \text { otherwise. } &
\end{array}\right.
$$

Moreover, all the nontrivial exotic 2-cocycles of $\mathfrak{r}_{\diamond}$ are nontrivial exotic 2cocycles of $\operatorname{Sym}_{0}\left(\mathcal{E}_{3}\right)$ as well. Therefore they define a non-central extension $\widehat{\mathfrak{r}}_{\diamond}$ of the Lie algebra $\mathfrak{r}_{\diamond}$ and hence a non-central extension $\mathfrak{r}_{2, \infty} \rtimes \widehat{\mathfrak{r}}_{\diamond}$ of the Lie 
algebra $\operatorname{Sym}_{0}\left(\mathcal{E}_{3}\right)$. The additional Maurer-Cartan forms $\beta_{7}, \ldots, \beta_{12}$ for the extended Lie algebra are solutions to system

$$
\left\{\begin{array}{l}
d \beta_{7}=\left(\beta_{1}+\beta_{3}\right) \wedge \beta_{7}+\beta_{2} \wedge \beta_{5} \\
d \beta_{8}=\beta_{1} \wedge \beta_{8}+\beta_{1} \wedge \beta_{2} \\
d \beta_{9}=\beta_{1} \wedge \beta_{9}+\beta_{2} \wedge \beta_{3} \\
d \beta_{10}=\left(\beta_{3}-\beta_{1}\right) \wedge \beta_{10}+\beta_{1} \wedge \beta_{4} \\
d \beta_{11}=\left(\beta_{3}-\beta_{1}\right) \wedge \beta_{11}+\beta_{3} \wedge \beta_{4} \\
d \beta_{12}=\left(3 \beta_{3}-2 \beta_{1}\right) \wedge \beta_{12}+\beta_{4} \wedge \beta_{6} .
\end{array}\right.
$$

This system is compatible with equations (36), (37).

Combining direct integration of the structure equations with Cartan's method of equivalence we get the explicit expressions for the Maurer-Cartan forms

$\beta_{1}=\frac{d a_{0}}{a_{0}}, \quad \beta_{2}=a_{0} d t, \quad \beta_{3}=\frac{d a_{1}}{a_{1}}, \quad \beta_{4}=\frac{2 a_{1} d s}{a_{0}}, \quad \beta_{5}=a_{1}(d z+2 s d t)$,

$\beta_{6}=\frac{a_{1}^{2}}{a_{0}}\left(d x+s d z+s^{2} d t\right), \quad \beta_{10}=\frac{a_{1}}{a_{0}}\left(d w+2 \ln a_{0} d s\right)$,

$\theta_{0}=b\left(d y+r_{0} d t\right), \quad \theta_{1}=\frac{a_{1} b}{a_{0}}\left(d x_{1}+p_{1} d y+r_{0} d z+r_{1} d t\right)$

while the linear combination

$\theta_{1}-\beta_{6}=\frac{a_{1} b_{0}}{a_{0}}\left(d x_{1}+p_{1} d y-b_{0}^{-1}\left(a_{1} d x+\left(a_{1} s-b_{0} r_{0}\right) d z+\left(a_{1} s^{2}-b_{0} r_{1}\right) d t\right)\right)$

must be a multiple of the contact form $d u-u_{t} d t-u_{x} d x-u_{y} d y-u_{z} d z$. Therefore we put $x_{1}=u, p_{1}=-u_{y}, a_{1}=b_{0} u_{x}, r_{0}=-u_{z}+s u_{x}, r_{1}=$ $-u_{t}+s^{2} u_{x}$.

Our attempts to find a linear combination of the Maurer-Cartan forms $\beta_{1}, \ldots, \beta_{12}, \theta_{k, i, j}$ have not given a Wahlquist-Estabrook form of any covering over equation (3) . Therefore we have extended the Lie algebra $\widehat{\mathfrak{r}}_{\diamond}$ with the structure equations (36), (38) via the same procedure, that is, by finding nontrivial 2-cocycles from $H_{c_{1} \beta_{1}+c_{2} \beta_{3}}^{2}\left(\widehat{\mathfrak{r}}_{\diamond}\right)$. Direct computations produce 18 such cocycles. The cocycles generate a 18-dimensional non-central extension of the Lie algebra $\widehat{\mathfrak{r}}_{\diamond}$. In what follows it is enough to consider one-dimensional 
extension of $\widehat{\mathfrak{r}}_{\diamond}$ generated by the nontrivial 2-cocycle $\beta_{4} \wedge \beta_{10}$ of the differential $d_{2 \beta_{3}-2 \beta_{1}}$. For the associated Maurer-Cartan form $\beta_{13}$ we have equation

$$
d \beta_{13}=2\left(\beta_{3}-\beta_{1}\right) \wedge \beta_{13}+\beta_{4} \wedge \beta_{10} .
$$

This equation is automatically compatible with system (36), (37), (38). Integration of equation (39) gives

$$
\beta_{13}=\frac{a_{1}^{2}}{a_{0}^{2}}(d v-2 w d s) .
$$

Consider the linear combination

$$
\begin{aligned}
& \mu_{1}=\beta_{13}+\beta_{5}-\beta_{6}-\theta_{0} \\
& =\frac{b_{0}^{2} u_{x}^{2}}{a_{0}^{2}}\left(d v-2 w d s-a_{0} d x-a_{0} b_{0}^{-1} u_{x}^{-2}\left(u_{x}\left(b_{0} s u_{x}-a_{0}\right) d z+a_{0} d y\right.\right. \\
& \left.\left.\quad+\left(b_{0} s^{2} u_{x}^{2}-a_{0}\left(u_{z}+s u_{x}\right)\right) d t\right)\right)
\end{aligned}
$$

and alter notation by substituting $a_{0}=v_{x}, b_{0}=v_{x}^{2} u_{x}^{-1} v_{y}^{-1}, w=\frac{1}{2} v_{s}$. This gives the Wahlquist-Estabrook form

$$
\begin{aligned}
\mu_{1}= & \frac{v_{x}^{2}}{u_{x}^{2} v_{y}^{2}}\left(d v-v_{s} d s-v_{y} d y-v_{x} d x-\left(s v_{x}-u_{x} v_{y}\right) d z\right. \\
& \left.-\left(s^{2} v_{x}-\left(u_{z}+s u_{y}\right) v_{y}\right) d t\right)
\end{aligned}
$$

for the Lax representation

$$
\left\{\begin{array}{l}
v_{z}=s v_{x}-u_{x} v_{y}, \\
v_{t}=s^{2} v_{x}-\left(u_{z}+s u_{y}\right) v_{y}
\end{array}\right.
$$

of equation (3). System (40) differs from the Lax representation (9) by notation. 


\subsection{Integrable hierarchy associated to $4 \mathrm{D} U H E$}

To find an integrable hierarchy associated to equation (3) we use the technique of subsections 3.4 and 4.4. Instead of the formal series (35) with $k \in\{0,1\}$ for fixed $n \geq 2$ consider the formal series

$$
\Theta=\sum_{k=0}^{n} \sum_{i=0}^{\infty} \sum_{j=0}^{\infty} h_{0}^{k} \frac{h_{1}^{i}}{i !} \frac{h_{2}^{j}}{j !} \theta_{k, i, j} .
$$

Then the crucial question is how to generalize system (37) for the series (41) (note, i.e., that system (37) does not involve the Maurer-Cartan form $\beta_{6}$ ). We propose to consider system

$$
\begin{aligned}
d \Theta= & \nabla_{2}(\Theta) \wedge \Theta+\nabla_{1}(\Theta) \wedge\left(\beta_{2}+h_{1} \beta_{1}+h_{0}\left(\beta_{5}+h_{1} \beta_{4}\right)+h_{0}^{2} \beta_{6}\right) \\
& +h_{0} \nabla_{0}(\Theta) \wedge\left(\beta_{1}-\beta_{3}+\frac{1}{2} \beta_{4}\right)-\frac{1}{2} \Theta \wedge \beta_{4}
\end{aligned}
$$

that includes (37) as a subsystem. To simplify notation in what follows we rename independent variables as $t=y_{2}, x=y_{0}, y=t_{0}$, and $z=y_{1}$. Integrating equations from (42) we get

$$
\begin{gathered}
\theta_{k}=\frac{a_{1}^{k} b}{a_{0}^{k}}\left(\sum_{i=0}^{k} P_{k i} d t_{k-i}+r_{n} d y_{2}+\left(\sum_{j=0}^{k-1}(-1)^{j} r_{k-1-j} s^{j}\right) d y_{1}\right. \\
\left.+\left(\sum_{j=1}^{k-1}(-1)^{j} j r_{k-1-j} s^{j-1}\right) d y_{0}\right)
\end{gathered}
$$

for $k \in\{0, \ldots, n\}$, where $r_{0}, \ldots, r_{n}$ are parameters, and polynomials $P_{k i}$ are defined by (25). We take the linear combination $\theta_{n}-\beta_{6}$, put $t_{n}=u$, solve the triangular linear system $P_{n i}=-u_{t_{n-i}}, i \in\{0, \ldots, n-1\}$, with respect to unknowns $p_{1}, p_{2}, \ldots, p_{n}$, then consequently put

$$
\begin{aligned}
& r_{n}=-u_{y_{2}}+a_{0}^{n-1} a_{1}^{2-n} b_{0}^{-1} s^{2}, \\
& r_{n-1}=-u_{y_{1}}-\sum_{j=1}^{n-1}(-1)^{j} r_{n-1-j} s^{j}+a_{0}^{n-1} a_{1}^{2-n} b_{0}^{-1} s, \\
& r_{n-2}= \begin{cases}-u_{y_{0}}-\sum_{j=2}^{n-1}(-1)^{j} j r_{n-1-j} s^{j-1}+a_{0}^{n-1} a_{1}^{2-n} b_{0}^{-1}, & n \geq 3, \\
-u_{y_{0}}+a_{0}^{n-1} a_{1}^{2-n} b_{0}^{-1}, & n=2,\end{cases}
\end{aligned}
$$


and finally rename $t_{i}=x_{n-1-i}, i \in\{0, \ldots, n-1\}$. This gives the contact form

$$
\theta_{n}-\beta_{6}=\frac{a_{1}^{n}}{a_{0}^{n}}\left(d u-\sum_{i=0}^{n-1} u_{x_{i}} d x_{i}-\sum_{j=0}^{2} u_{y_{j}} d y_{j}\right) .
$$

Now we take the linear combination $\mu_{n}=\beta_{13}+\beta_{5}-\beta_{6}-\theta_{n-1}$, rename $b=a_{0}^{n-3} a_{1}^{3-n} v_{x_{0}}$, and put

$$
a_{0}= \begin{cases}v_{y_{0}}-v_{x_{0}} \sum_{j=0}^{n-3}(-1)^{j}(j+1) r_{n-3-j} s^{j}, & n \geq 3 \\ v_{y_{0}}, & n=2 .\end{cases}
$$

Thus we obtain

$$
\begin{aligned}
\mu_{n}= & \frac{a_{1}^{2}}{a_{0}^{2}}\left(d v-v_{s} d s-v_{y_{0}}\left(d y_{0}+s d y_{1}+s^{2} d y_{2}\right)-v_{x_{0}}\left(u_{y_{0}} d y_{1}+\left(u_{y_{1}}+s u_{y_{0}}\right) d y_{2}\right)\right. \\
& \left.-v_{x_{0}} d x_{0}-\sum_{i=0}^{n-1}\left(s^{i}-\sum_{j=0}^{i-1} s^{i-j-1} u_{x_{j}}\right) v_{x_{0}} d x_{i}\right) .
\end{aligned}
$$

This is the Wahlquist-Estabrook form for the Lax representation

$$
\left\{\begin{aligned}
v_{y_{1}}= & s v_{y_{0}}-u_{y_{0}} v_{x_{0}} \\
v_{y_{2}}= & s^{2} v_{y_{0}}-\left(u_{y_{1}}+s u_{y_{0}}\right) v_{x_{0}} \\
v_{x_{1}}= & \left(s-u_{x_{0}}\right) v_{x_{0}} \\
v_{x_{2}}= & \left(s^{2}-s u_{x_{0}}-u_{x_{1}}\right) v_{x_{0}} \\
& \cdots \\
v_{x_{i}}= & \left(s^{i}-\sum_{j=0}^{i-1} s^{i-j-1} u_{x_{j}}\right) v_{x_{0}} \\
& \cdots \\
v_{x_{n-1}}= & \left(s^{n-1}-s^{n-2} u_{x_{0}}-s^{n-3} u_{x_{1}}-\ldots-s u_{x_{n-3}}-u_{x_{n-2}}\right) v_{x_{0}} .
\end{aligned}\right.
$$

Equations for $v_{x_{i}}$ coincide with system (26). The compatibility conditions of system (43) define the integrable hierarchy associated to equation (3). This hierarchy includes system $\mathcal{H}_{n-1}$, equation (3) written as (24), and system

$u_{x_{k} y_{0}}=u_{x_{k-2} y_{2}}+u_{y_{1}} u_{x_{0} x_{k-2}}-u_{x_{k-2}} u_{x_{0} y_{1}}+u_{y_{0}} u_{x_{0} x_{k-1}}-u_{x_{k-1}} u_{x_{0} y_{0}}$,

$u_{x_{m} y_{1}}=u_{x_{m-1} y_{2}}+u_{y_{1}} u_{x_{0} x_{m-1}}-u_{x_{m-1}} u_{x_{0} y_{1}}$,

where $k \in\{2, \ldots, n-1\}, m \in\{1, \ldots, n-1\}$, and $u_{x_{0} x_{i}}$ are replaced by the right-hard sides of equations from $\mathcal{H}_{n-1}$. System (43) can be included in the construction of [7]. 


\section{The four-dimensional Martínez Alonso-Shabat equation}

Equation (44) does not belong to the hierarchies from the previous sections. Its Lax representation (10) was found in [33] via the method of [31]. In this section we show that (10) can be constructed by the technique of the present paper as well. Also we find the integrable hierarchy generated by the natural extensions of the symmetry algebra $\operatorname{Sym}_{0}\left(\mathcal{E}_{4}\right)$ of equation (4).

\subsection{Contact symmetries}

The Lie algebra $\operatorname{Sym}_{0}\left(\mathcal{E}_{4}\right)$ is generated by symmetries

$$
V_{0}(A)=-A u_{x}+A_{x} u-A_{t} z, \quad V_{1}(A)=A, \quad W(B)=-B u_{y},
$$

that depend on arbitrary functions $A=A(t, x), B=B(y, z)$, and symmetries

$$
X_{1}=-u_{t}, \quad X_{2}=-t u_{t}, \quad X_{3}=-u_{z}, \quad X_{4}=-z u_{z} .
$$

The commutator table

$$
\begin{array}{ll}
\left\{V_{i}(A), V_{j}(\tilde{A})\right\}=V_{i+j}\left(A \tilde{A}_{x}-\tilde{A} A_{x}\right), & \left\{V_{i}(A), W(B)\right\}=0, \\
\{W(B), W(\tilde{B})\}=W\left(B \tilde{B}_{y}-\tilde{B} B_{y}\right), & \left\{X_{1}, V_{0}(A)\right\}=V_{0}\left(A_{t}\right), \\
\left\{X_{2}, V_{0}(A)\right\}=V_{0}\left(t A_{t}\right), & \left\{X_{3}, V_{0}(A)\right\}=-V_{1}\left(A_{t}\right), \\
\left\{X_{4}, V_{0}(A)\right\}=0, & \left\{X_{1}, V_{1}(A)\right\}=V_{1}\left(A_{t}\right), \\
\left\{X_{2}, V_{1}(A)\right\}=V_{1}\left(t A_{t}+A\right), & \left\{X_{3}, V_{1}(A)\right\}=0, \\
\left\{X_{4}, V_{1}(A)\right\}=-V_{1}(A), & \left\{X_{1}, W(B)\right\}=0, \\
\left\{X_{2}, W(B)\right\}=0, & \left\{X_{3}, W(B)\right\}=W\left(B_{z}\right), \\
\left\{X_{4}, W(B)\right\}=W\left(z B_{z}\right), & \left\{X_{1}, X_{2}\right\}=X_{1}, \\
\left\{X_{1}, X_{3}\right\}=0, & \left\{X_{1}, X_{4}\right\}=0, \\
\left\{X_{2}, X_{3}\right\}=0, & \left\{X_{2}, X_{4}\right\}=0, \\
\left\{X_{3}, X_{4}\right\}=X_{3} . &
\end{array}
$$

implies that $\operatorname{Sym}_{0}\left(\mathcal{E}_{4}\right)=\mathfrak{s}_{2,1}=\mathfrak{s}_{2,1, \infty} \rtimes \mathfrak{s}_{\diamond}$, where $\mathfrak{s}_{2,1, \infty}=\left(\mathbb{R}_{2}\left[h_{0}\right] \otimes \mathfrak{w}[t, x]\right) \oplus$ $\mathfrak{w}[z, y]$ and $\mathfrak{s}_{\diamond}=\left\langle X_{1}, X_{2}\right\rangle \oplus\left\langle X_{3}, X_{4}\right\rangle$ (direct sums of Lie algebras).

\subsection{Maurer-Cartan forms and the second exotic cohomology group}

Let the Maurer-Cartan forms $\theta_{k, i, j}, \omega_{i j}, \alpha_{0}, \alpha_{1}, \beta_{0}, \beta_{1}$ be defined by requirement that there hold $\theta_{k, i, j}\left(V_{k^{\prime}}\left(t^{i^{\prime}} x^{j^{\prime}}\right)\right)=i ! j ! \delta_{k k^{\prime}} \delta_{i i^{\prime}} \delta_{j j^{\prime}}, \omega_{i, j}\left(W\left(y^{i^{\prime}} z^{j^{\prime}}\right)\right)=$ $\delta_{i i^{\prime}} \delta_{j j^{\prime}}, \alpha_{m}\left(X_{m^{\prime}+1}\right)=\delta_{m m^{\prime}}, \beta_{m}\left(X_{m^{\prime}+3}\right)=\delta_{m m^{\prime}}$, while all the other values of 
these forms on the generators of $\operatorname{Sym}_{0}\left(\mathcal{E}_{4}\right)$ are equal to zero. Then the structure equations for $\operatorname{Sym}_{0}\left(\mathcal{E}_{4}\right)$ are given by systems

$$
\left\{\begin{array}{l}
d \alpha_{0}=0 \\
d \alpha_{1}=\alpha_{0} \wedge \alpha_{1} \\
d \beta_{0}=0 \\
d \beta_{1}=\beta_{0} \wedge \beta_{1}
\end{array}\right.
$$

and

$$
\left\{\begin{aligned}
d \Theta= & \nabla_{2}(\Theta) \wedge \Theta+\nabla_{1}(\Theta) \wedge\left(\alpha_{1}+h_{1} \alpha_{0}-h_{0} \beta_{1}\right) \\
& +h_{0} \nabla_{0}(\Theta) \wedge\left(\alpha_{0}-\beta_{0}\right) \\
d \Omega= & \nabla_{3}(\Omega) \wedge \Omega+\nabla_{4}(\Omega) \wedge\left(\beta_{1}+h_{4} \beta_{0}\right) .
\end{aligned}\right.
$$

where

$$
\Theta=\sum_{k=0}^{1} \sum_{i=0}^{\infty} \sum_{j=0}^{\infty} h_{0}^{k} \frac{h_{1}^{i}}{i !} \frac{h_{2}^{j}}{j !} \theta_{k, i, j}, \quad \Omega=\sum_{i=0}^{\infty} \sum_{j=0}^{\infty} \frac{h_{3}^{i} h_{4}^{j}}{i ! j !} \omega_{i, j} .
$$

System (44) defines the structure equations for the Lie algebra $\mathfrak{s}_{\diamond}$. Direct computations show that the following statement holds.

Proposition 4. $H^{1}\left(\operatorname{Sym}_{0}\left(\mathcal{E}_{4}\right)\right)=\left\langle\alpha_{0}, \beta_{0}\right\rangle$,

$$
H_{c_{1} \alpha_{0}+c_{2} \beta_{0}}^{2}\left(\mathfrak{s}_{\diamond}\right)= \begin{cases}\left\langle\left[\alpha_{0} \wedge \alpha_{1}\right],\left[\beta_{0} \wedge \alpha_{1}\right]\right\rangle, & c_{1}=1, c_{2}=0, \\ \left\langle\left[\alpha_{0} \wedge \beta_{1}\right],\left[\beta_{0} \wedge \beta_{1}\right]\right\rangle, & c_{1}=0, c_{2}=1, \\ \left\langle\left[\alpha_{1} \wedge \beta_{1}\right]\right\rangle, & c_{1}=1, c_{2}=1, \\ \{[0]\}, & \text { otherwise, }\end{cases}
$$

and $H_{c_{1} \alpha_{0}+c_{2} \beta_{0}}^{2}\left(\mathfrak{s}_{\diamond}\right) \subseteq H_{c_{1} \alpha_{0}+c_{2} \beta_{0}}^{2}\left(\operatorname{Sym}_{0}\left(\mathcal{E}_{4}\right)\right)$ for $\left(c_{1}, c_{2}\right)=(1,0),\left(c_{1}, c_{2}\right)=$ $(0,1),\left(c_{1}, c_{2}\right)=(1,1)$. Equations

$$
\left\{\begin{array}{l}
d \gamma_{1}=\alpha_{0} \wedge \gamma_{1}+\alpha_{0} \wedge \alpha_{1} \\
d \gamma_{2}=\alpha_{0} \wedge \gamma_{2}+\beta_{0} \wedge \alpha_{1} \\
d \gamma_{3}=\beta_{0} \wedge \gamma_{3}+\alpha_{0} \wedge \beta_{1} \\
d \gamma_{4}=\beta_{0} \wedge \gamma_{4}+\beta_{0} \wedge \beta_{1} \\
d \gamma_{5}=\left(\alpha_{0}+\beta_{0}\right) \wedge \gamma_{5}+\alpha_{1} \wedge \beta_{1}
\end{array}\right.
$$

with unknown 1-forms $\gamma_{1}, \ldots, \gamma_{5}$ are compatible with the structure equations (44), (45) of $\operatorname{Sym}_{0}\left(\mathcal{E}_{4}\right)$. System (44), (45), (47) defines five-dimensional non-central extension for this Lie algebra. 


\subsection{Lax representation of the $4 D$ MASh equation}

Integration of the structure equations (44), (45), (47) gives the MaurerCartan forms $\alpha_{0}=d b_{1} / b_{1}, \alpha_{1}=b_{1} d t, \beta_{0}=d b_{2} / b_{2}, \beta_{1}=b_{2} d z, \gamma_{1}=b_{1}\left(d v_{1}+\right.$ $\left.\ln b_{1} d t\right), \gamma_{2}=b_{1}\left(d v_{2}+\ln b_{2} d t\right), \gamma_{3}=b_{2}\left(d v_{3}+\ln b_{1} d z\right), \gamma_{4}=b_{2}\left(d v_{4}+\ln b_{2} d z\right)$, $\theta_{0}=a_{1}\left(d x+q_{0} d t\right), \theta_{1}=a_{1} b_{2}\left(d x_{1}+p_{1} d x-q_{0} d z+q_{1} d t\right), \omega=\omega_{0,0}=$ $a_{2}\left(d y+s_{0} d t\right)$, where $b_{1}>0, b_{2}>0, a_{1} \neq 0, a_{2} \neq 0$. The results of Cartan's method of equivalence show that the linear combination $\theta_{1}-\omega$ is a multiple of the contact form $d u-u_{t} d t-u_{x} d x-u_{y} d y-u_{z} d z$. Therefore we put $x_{1}=u$, $p_{1}=-u_{x}, q_{1}=-u_{t}, a_{2}=a_{1} b_{2} b_{1}^{-1} u_{y}, q_{0}=u_{z}-s_{0} u_{y}$. Then we consider the linear combination

$$
\begin{aligned}
& \mu_{1}=\gamma_{4}-\gamma_{3}+\alpha_{1}-\beta_{1}-\theta_{0}-\omega \\
& =b_{2}\left(d v_{4}-d v_{3}-a_{1} b_{2}^{-1} d x-a_{1} b_{2}^{-1}\left(u_{z}-s_{0} u_{y}\right) d t\right. \\
& \left.\quad \quad-a_{1} b_{1}^{-1} u_{y} d y-\left(a_{1} b_{1}^{-1} s_{0} u_{y}+\ln b_{1}-\ln b_{2}\right) d z+b_{1}^{-1} b_{2}^{-1} d b_{1}-b_{2}^{-2} d b_{2}\right) .
\end{aligned}
$$

We substitute $v_{4}=v+v_{3}, b_{2}=s b_{1}, a_{1}=b_{1} s v_{x}, s_{0}=u_{y}^{-1}\left(u_{z}-v_{t} v_{x}^{-1}\right)$, and then put $b_{1}=s^{-2} v_{s}^{-1}$. This yields the Wahlquist-Estabrook form

$\mu_{1}=\frac{1}{s v_{s}}\left(d v-v_{s} d s-v_{t} d t-v_{x} d x-s u_{y} v_{x} d y-\left(s\left(u_{z} v_{x}-v_{t}\right)-\ln s\right) d z\right)$.

of the Lax representation

$$
\left\{\begin{array}{l}
v_{y}=s u_{y} v_{x} \\
v_{z}=s\left(u_{z} v_{x}-v_{t}\right)-\ln s
\end{array}\right.
$$

for equation (44). The change of variables $s \mapsto \lambda, v \mapsto v-z \ln \lambda$, transforms system (48) to the form (10).

\subsection{Integrable hierarchy associated to $4 D$ MASh equation}

Consider a series of natural extensions

$$
\mathfrak{s}_{n, m}=\left(\mathbb{R}_{n}\left[h_{0}\right] \otimes \mathfrak{w}[t, x]\right) \oplus\left(\mathbb{R}_{m}\left[h_{0}\right] \otimes \mathfrak{w}[z, y]\right) \rtimes \mathfrak{s}_{\diamond}
$$

of the Lie algebra $\operatorname{Sym}_{0}\left(\mathcal{E}_{4}\right)=\mathfrak{s}_{2,1}$. We replace the series for $\Theta$ from (46) in (45) by

$$
\Theta=\sum_{k=0}^{n} \sum_{i=0}^{\infty} \sum_{j=0}^{\infty} h_{0}^{k} \frac{h_{1}^{i}}{i !} \frac{h_{2}^{j}}{j !} \theta_{k, i, j}
$$


Then the key question there is how to generalize equations (45) for the series

$$
\Omega=\sum_{k=0}^{m} \sum_{i=0}^{\infty} \sum_{j=0}^{\infty} h_{0}^{k} \frac{h_{3}^{i}}{i !} \frac{h_{4}^{j}}{j !} \omega_{k, i, j}
$$

instead of the series for $\Omega$ from (46).

We propose to define the Lie algebra $\mathfrak{s}_{n, m}$ by the structure equations that include system (44) and system

$$
\left\{\begin{aligned}
d \Theta= & \nabla_{2}(\Theta) \wedge \Theta+\nabla_{1}(\Theta) \wedge\left(\alpha_{1}+h_{1} \alpha_{0}-h_{0} \beta_{1}\right) \\
& +h_{0} \nabla_{0}(\Theta) \wedge\left(\alpha_{0}-\beta_{0}\right) \\
d \Omega= & \nabla_{3}(\Omega) \wedge \Omega+\nabla_{4}(\Omega) \wedge\left(\beta_{1}+h_{4} \beta_{0}-h_{0} \alpha_{1}\right) \\
& +h_{0} \nabla_{0}(\Omega) \wedge\left(\beta_{0}-\alpha_{0}\right)
\end{aligned}\right.
$$

with $\Theta$ and $\Omega$ given by (49) and (50). We have $H_{c_{1} \alpha_{0}+c_{2} \beta_{0}}^{2}\left(\mathfrak{s}_{\diamond}\right) \subseteq H_{c_{1} \alpha_{0}+c_{2} \beta_{0}}^{2}\left(\mathfrak{s}_{n, m}\right)$ for $\left(c_{1}, c_{2}\right)=(1,0),\left(c_{1}, c_{2}\right)=(0,1),\left(c_{1}, c_{2}\right)=(1,1)$, therefore system (47) defines a non-central extension of the Lie algebra $\mathfrak{s}_{n, m}$.

For simplicity of computations we take $m=n$ in what follows, also we rename the independent variables as $t=t_{0}, x=x_{0}$. Then we find explicit expressions for the Maurer-Cartan forms $\theta_{k}=\theta_{k, 0,0}, \omega_{k}=\omega_{k, 0,0}, k \in\{0, \ldots, n\}$ with fixed $n \geq 3$.

Integrating the structure equations (51) we obtain

$$
\begin{aligned}
& \theta_{k}=a_{1} b_{1}^{-k} b_{2}^{k}\left(d x_{k}+\sum_{i=1}^{k} p_{i} d x_{k-i}-q_{k-1} d z+q_{n} d t\right), \\
& \omega_{k}=a_{2} b_{1}^{k} b_{2}^{-k}\left(d y_{k}+\sum_{i=1}^{k} r_{i} d y_{k-i}-s_{k-1} d t+q_{n} d z\right),
\end{aligned}
$$

where $p_{i}, q_{i}, r_{i}, s_{i} \in \mathbb{R}$ are free parameters. Then we put consequently $x_{n}=u$, $b_{2}=b_{1} s, p_{1}=-u_{x_{n-1}}+s^{-1}, p_{k}=-u_{x_{n-k}}+2^{k-1} s^{-k}-\sum_{j=1}^{k-1} 2^{j-1} s^{-j} u_{x_{n+j-k}}$, for $k \in\{2, \ldots, n\}, r_{1}=u_{y_{n-2}} u_{y_{n-1}}^{-1}-s, r_{k}=\left(u_{y_{n-k-1}}-s u_{y_{n-k}}\right) u_{y_{n-1}}^{-1}$ for $k \in\{2, \ldots, n-1\}, q_{n}=-u_{t}+s^{-1}\left(-u_{z}+2 q_{n-1}+s_{n-1} u_{y_{n-1}}\right)$, and $q_{0}=-s^{n-1}\left(u_{z}-u_{y_{n-1}} \sum_{j=0}^{n-1} s^{n-1-j} s_{j}\right)+s^{n-1} q_{n-1}-\sum_{k=0}^{n-2} s^{k} q_{k}$. This yields the contact form

$$
\theta_{n}-\sum_{k=0}^{n-1}\left(\theta_{k}+\omega_{k}\right)=a_{1} b_{1}^{-n} b_{2}^{-n}\left(d u-\sum_{k=1}^{n-1}\left(u_{t_{k}} d t_{i}+u_{x_{k}} d x_{k}\right)-u_{y} d y-u_{z} d z\right) \text {. }
$$


Then we consider the linear combination

$$
\mu_{n}=\gamma_{4}-\gamma_{3}+\alpha_{0}-\beta_{0}-\theta_{n-1}+\sum_{k=0}^{n-3}(n-k-2) \theta_{k}-\sum_{k=0}^{n-1}(n-k) \omega_{k}
$$

and put consequently $v_{4}=v+v_{3}, a_{1}=b_{1} s^{2-n} v_{x_{n-1}}, b_{1}=s^{-2} v_{s}$,

$$
\begin{gathered}
q_{1}=s^{n-2}\left(v_{t} v_{x_{n-1}}^{-1}-(n-2) u_{z}+(n-3) s_{n-1}\right)-\sum_{k=2}^{n-2} k s^{k-1} q_{k} \\
+u_{y_{n-1}} \sum_{k=0}^{n-1}(2 n-k-3) s^{2 n-k-3} s_{k}
\end{gathered}
$$

in the case $n>3$ and

$$
q_{1}=s\left(v_{t} v_{x_{2}}^{-1}-u_{z}\right)+u_{y_{2}} \sum_{k=0}^{2}(3-k) s^{3-k} s_{k}
$$

when $n=3$. Finally we rename $x_{k} \mapsto x_{n-k-1}, y_{k} \mapsto y_{n-k-1}$ for $k \in\{0, \ldots, n-$ $1\}$. This gives the Wahlquist-Estabrook form

$$
\begin{aligned}
\mu_{n}= & \frac{1}{s v_{s}}\left(d v-v_{s} d s-v_{t} d t-v_{x_{0}} d x_{0}-\left(s\left(u_{z} v_{x_{0}}-v_{t}\right)-\ln s\right) d z\right. \\
& \left.-\sum_{k=0}^{n-1}\left(\sum_{j=0}^{k} s^{k-j} u_{y_{j}}\right) v_{x_{0}} d y_{k}-\sum_{m=1}^{n-1}\left(s^{-m}-\sum_{j=0}^{m-1} s^{1-m-j} u_{x_{j}}\right) v_{x_{0}} d x_{m}\right)
\end{aligned}
$$


for the Lax representation

$$
\left\{\begin{aligned}
v_{x_{1}}= & \left(s^{-1}-u_{x_{0}}\right) v_{x_{0}} \\
& \cdots \\
v_{x_{m}}= & \left(s^{-m}-\sum_{j=0}^{m-1} s^{1-m-j} u_{x_{j}}\right) v_{x_{0}} \\
& \cdots \\
v_{x_{n-1}}= & \left(s^{1-n}-s^{2-n} u_{x_{0}}-s^{3-n} u_{x_{1}}-\ldots-u_{x_{n-2}}\right) v_{x_{0}} \\
v_{y_{0}}= & s u_{y_{0}} v_{x_{0}} \\
& \cdots \\
v_{y_{k}}= & \left(\sum_{j=0}^{k} s^{k+1-j} u_{y_{j}}\right) v_{x_{0}} \\
& \cdots \\
v_{y_{n-1}}= & \left(\sum_{j=0}^{k} u_{y_{n-1}}+s u_{y_{n-2}}+\ldots+s^{n-2} u_{y_{0}}\right) v_{x_{0}} \\
v_{z}= & s\left(u_{z} v_{x_{0}}-v_{t}\right)-\ln s .
\end{aligned}\right.
$$

Equations for $v_{x_{m}}$ differ from system (26) by the change $s \mapsto s^{-1}$. The compatibility conditions of system (52) define the integrable hierarchy associated to equation (4). This hierarchy includes system $\mathcal{H}_{n-1}$ as well as equation (4) written in the form

$$
u_{t y_{0}}=u_{z} u_{x_{0} y_{0}}-u_{y_{0}} u_{x_{0} z}
$$

systems

$$
\begin{aligned}
& u_{t x_{k}}=-u_{x_{k+1} z}+u_{z} u_{x_{0} x_{k}}-u_{x_{k}} u_{x_{0} z}, \\
& u_{y_{m}}=-u_{y_{m-1} z}+u_{z} u_{x_{0} y_{m}}-u_{y_{m}} u_{x_{0} z}, \\
& u_{x_{k+1} y_{0}}=u_{y_{0}} u_{x_{0} x_{k}}-u_{x_{k}} u_{x_{0} y_{0}}, \\
& u_{x_{k+1} y_{m}}=u_{x_{k} y_{m-1}}+u_{y_{m}} u_{x_{0} x_{k}}-u_{x_{0}} u_{x_{k} y_{m}} \\
& u_{y_{0} y_{k}}=u_{y_{0}} u_{x_{0} y_{k+1}}-u_{y_{k+1}} u_{x_{0} y_{0}}
\end{aligned}
$$

with $k \in\{0, \ldots, n-2\}, m \in\{1, \ldots, n-1\}$, and system

$$
u_{y_{i} y_{j}}=u_{y_{i-1} y_{j+1}}+u_{y_{i}} u_{x_{0} y_{j+1}}-u_{y_{j+1}} u_{x_{0} y_{i}}
$$


with $i \in\{1, \ldots, n-2\}, j \in\{i, \ldots, n-2\}$.

Some equations from systems (53) - (58) differ by notation from equations (21) - (66). Equations (53), (56) with $k=0$, equation (58) with $i=j=1$, and equations (55), (157) with $k>0$ correspond to equations (2), (3), and (44), respectively. Equations (53) with $k>0$ or equations (54), and equation (57) with $k=0$ agree with equations (51) and (6), respectively. Equation (55) with $k=0$ is the 3-dimensional rdDym equation, [4, 39, 31, 3], When the right-hand sides of equations (56), (58) inlcude the terms $u_{x_{k} y_{m-1}}$, $u_{x_{k} y_{m}}$, and $u_{y_{i-1} y_{j+1}}$ from the left-hand sides of equations from the same systems, these terms have to be replaced using the corresponding equations. This yields equations of increasing dimensions.

\section{Conclusion}

In the present paper we have shown that the method of [34, 35] is applicable to Lax representations with non-removable parameters, in particular, the Lax representations for equations (11) - (4) can be derived from the noncentral extensions of contact symmetry algebras of these equations. In all the examples the symmetry algebras have the specific structure of the semi-direct product of an infinite-dimensional ideal and a non-Abelian finite-dimensional Lie subalgebra. The cohomological properties of the finite-dimensional subalgebras turn out to be sufficient to reveal non-central extensions that define the Lax representations.

For the considered equations the infinite-dimensional ideals of the symmetry algebras are either of the form of tensor products of the algebra of truncated polynomials with an infinite-dimensional Lie algebra, or contains such tensor products as direct summands. The natural procedure of increasing the degree of the truncated polynomials produces a series of natural extensions of the symmetry algebras of the PDEs under the study. These extensions inherit the nontrivial exotic 2-cocycles and thus admit non-central extensions generated by these cocycles. We have shown that this procedure gives integrable hierarchies associated with equations (1), (2), (3), and (4).

It is natural to ask whether the method is be applicable in the case when the symmetry algebra of the PDE has more complicated structure. Also, we expect that the proposed technique will be helpful in describing multicomponent integrable generalizations of integrable PDEs, [14, 24, 6, 32, 22]. We intend to address these issues in the further study. 


\section{Acknowledgments}

This work was partially supported by the Faculty of Applied Mathematics of AGH UST statutory tasks within subsidy of Ministry of Science and Higher Education.

I am very grateful to I.S. Krasil'shchik for useful discussions. I thank L.V. Bogdanov, M.V. Pavlov, and P. Zusmanovich for important remarks.

\section{References}

\section{References}

[1] M.J. Ablowitz, P.A. Clarkson. Solitons, Nonlinear Evolution Equations and Inverse Scattering. Cambridge University Press, Cambridge, 1991

[2] H. Baran, I.S. Krasil'shchik, O.I. Morozov, P. Vojčák. Fivedimensional Lax-integrable equation, its reductions and recursion operator. Lobachevskii J. of Math., 36 (2015) 225-233

[3] H. Baran, I.S. Krasil'shchik, O.I. Morozov, P. Vojčák. Coverings over Lax integrable equations and their nonlocal symmetries. Theor. Math. Phys., 188 (2016), 1273-1295

[4] Błaszak M. Classical R-matrices on Poisson algebras and related dispersionless systems. Phys. Lett. A, 297 (2002), 191-195

[5] H. Baran, M. Marvan. Jets: A software for differential calculus on jet spaces and diffieties. Available on-line at http://jets.math.slu.cz

[6] L.V. Bogdanov. Non-Hamiltonian generalizations of the dispersionless 2DTL hierarchy. J. Phys. A: Math. Theor. 43 (2010), 434008

[7] L.V. Bogdanov, M.V. Pavlov. Linearly degenerate hierarchies of quasiclassical SDYM type. J. Math. Phys. 58 (2017), 093505

[8] Cartan É. Sur la structure des groupes infinis de transformations. In: Cartan É., Euvres Complètes, Part II, 2, 571-715. Gauthier - Villars, Paris, 1953

[9] Cartan É. Les sous-groupes des groupes continus de transformations. In: Cartan É., Euvres Complètes, Part II, 2, 719-856. Gauthier - Villars, Paris, 1953 
[10] Cartan É. Les problèmes d'équivalence. In: Cartan É., (Euvres Complètes, Part II, 2, 1311-1334. Gauthier - Villars, Paris, 1953

[11] Cartan É. La structure des groupes infinis. In: Cartan É., (Euvres Complètes, Part II, 2, 1335-1384. Gauthier - Villars, Paris, 1953

[12] A. Das. Integrable Models. World Scientific, Singapore, 1989.

[13] R. Dodd, A. Fordy. The prolongation structures of quasi-polynomial flows. Proc. R. Soc. London A 385 (1983), 389-429

[14] M. Dunajski. Anti-self-dual fourmanifolds with a parallel real spinor. Proc. Roy. Soc. Lond. A,458 (2002), 1205-1222

[15] M. Dunajski. A class of Einstein-Weil spaces associated to an integrable system of hydrodynamic type. J. Geom. Phys. 51 (2004) 126-137

[16] M. Fels, P.J. Olver. Moving coframes. I. A practical algorithm. Acta. Appl. Math. 51 (1998), 161-213

[17] Ferapontov E.V., Khusnutdinova K.R. Hydrodynamic reductions of multi-dimensional dispersionless PDEs: the test for integrability. J. Math. Phys., 45 (2004), 2365-2377

[18] J. Krasil'shchik, A. Verbovetsky. Geometry of jet spaces and integrable systems// J. Geom. Phys. 61 (2011), 1633-1674

[19] J. Krasil'shchik, A. Verbovetsky, R. Vitolo. A unified approach to computation of integrable structures. Acta Appl. Math. 120 (2012), 199-218

[20] I.S. Krasil'shchik, A.M. Vinogradov. Nonlocal symmetries and the theory of coverings, Acta Appl. Math. 2 (1984), 79-86

[21] I.S. Krasil'shchik, A.M. Vinogradov. Nonlocal trends in the geometry of differential equations:

symmetries, conservation laws, and Bäcklund transformations. Acta Appl. Math. 15 (1989), 161-209

[22] B.S. Kruglikov, O.I. Morozov. Integrable dispersionless PDEs in 4D, their symmetry pseudogroups and deformations. Lett. Math. Phys. 105 (2015), 1703-1723 
[23] G.M. Kuz'mina. On a possibility to reduce a system of two first-order partial differential equations to a single equation of the second order. Proc. Moscow State Pedagog. Inst. 271 (1967), 67-76 (in Russian)

[24] S.V. Manakov, P.M. Santini. Inverse scattering problem for vector fields and the Cauchy problem for the heavenly equation. Phys. Lett. A 359 (2006), 613

[25] S.V. Manakov, P.M. Santini. Integrable dispersionless PDEs arising as commutation condition of pairs of vector fields. J. Phys: Conf. Ser. 482 (2014), 012029

[26] L. Martínez Alonso, A.B. Shabat. Energy-dependent potentials revisited: A universal hierarchy of hydrodynamic type. Phys. Lett. A 299 (2002), 359-365

[27] L. Martínez Alonso, A.B. Shabat. Hydrodynamic reductions and solutions of a universal hierarchy. Theor. Math. Phys. 140 (2004), 1073-1085

[28] V.G. Mikhalev. On the Hamiltonian formalism for Kortewegde Vries type hierarchies. Functional Analysis and Its Applications, 26 No 2 (1992), 140-142

[29] O.I. Morozov. Moving coframes and symmetries of differential equations. J. Phys. A 35 (2002), 2965-2977

[30] O.I. Morozov. Contact-equivalence problem for linear hyperbolic equations. J. Math. Sci. 135 (2006), 2680-2694

[31] O.I. Morozov. Contact integrable extensions of symmetry pseudo-groups and coverings of $(2+1)$ dispersionless integrable equations. J. Geom. Phys. 59 (2009), 1461 - 1475

[32] O.I. Morozov. A two-component generalization of the integrable rdDym equation. SIGMA 8 (2012), 051

[33] O.I. Morozov. The four-dimensional Martínez Alonso-Shabat equation: Differential coverings and recursion operators J. Geom. Phys. 85 (2014), $75-80$

[34] O.I. Morozov. Deformed cohomologies of symmetry pseudo-groups and coverings of differential equations. J. Geom. Phys. 113 (2017), 215-225 
[35] O.I. Morozov. Deformations of infinite-dimensional Lie algebras, exotic cohomology, and integrable nonlinear partial differential equations. J. Geom. Phys. 128 (2018), 20-31

[36] S.P. Novikov. On exotic De-Rham cohomology. Perturbation theory as a spectral sequence. arXiv:math-ph/0201019, 2002

[37] S.P. Novikov. On metric-independent exotic homology. Proc. Steklov Inst. Math. 251 (2005), 206-212

[38] P.J. Olver. Equivalence, Invariants, and Symmetry Cambridge University Press, Cambridge, 1995

[39] M.V. Pavlov. Integrable hydrodynamic chains. J. Math. Phys. 44 (2003) 4134-4156

[40] M.V. Pavlov, N. Stoilov. Three dimensional reductions of fourdimensional quasilinear systems. J. Math. Phys. 58 (2017), 111510

[41] L.A. Takhtadzhyan, L.D. Faddeev. Hamiltonian Methods in the Theory of Solitons Springer, Berlin, 1987

[42] A.M. Vinogradov, I.S. Krasil'shchik (eds.) Symmetries and Conservation Laws for Differential Equations of Mathematical Physics [in Russian], Moscow: Factorial, 2005; English transl. prev. ed.: I.S. Krasil'shchik, A.M. Vinogradov (eds.) Symmetries and Conservation Laws for Differential Equations of Mathematical Physics. Transl. Math. Monogr., 182, Amer. Math. Soc., Providence, RI, 1999 